REVIEW ARTICLE

DOI: $10.1038 / \mathrm{s} 41467-018-04813-5$

\title{
Advances in highly doped upconversion nanoparticles
}

\author{
Shihui Wen ${ }^{1}$, Jiajia Zhou (1) ${ }^{1}$, Kezhi Zheng ${ }^{2}$, Artur Bednarkiewicz (iD) ${ }^{3,4}$ \\ Xiaogang Liu (iD ${ }^{2} \&$ Dayong Jin (D) ${ }^{1}$
}

Lanthanide-doped upconversion nanoparticles (UCNPs) are capable of converting near-infrared excitation into visible and ultraviolet emission. Their unique optical properties have advanced a broad range of applications, such as fluorescent microscopy, deep-tissue bioimaging, nanomedicine, optogenetics, security labelling and volumetric display. However, the constraint of concentration quenching on upconversion luminescence has hampered the nanoscience community to develop bright UCNPs with a large number of dopants. This review surveys recent advances in developing highly doped UCNPs, highlights the strategies that bypass the concentration quenching effect, and discusses new optical properties as well as emerging applications enabled by these nanoparticles. with lanthanide ions featuring a wealth of electronic transitions within the $4 f$ electron shells. These nanoparticles can up-convert two or more lower-energy photons into one high-energy photon ${ }^{1-3}$. Over the past decade, this unique anti-Stokes optical property has enabled a broad range of applications, spanning from background-free biological sensing and light-triggered drug delivery to solar energy harvesting and super-resolution microscopy ${ }^{4-6}$. To achieve high upconversion efficiency, it is essential to co-dope sensitizer ions alongside activator ions that have a closely matched intermediate-excited state ${ }^{7-9}$. This doping process requires a rational design that offers optimal interactions of a network of the sensitizer and activator ions, and the upconversion efficiency is highly dependent on the separating distance between the dopants. Therefore, the proper management of the doping concentration in a given nanoparticle will be the deciding factor in leveraging the energy transfer process and ultimately the luminescence performance of the nanoparticle ${ }^{9-11}$.

In stark contrast to quantum dots, UCNPs contain individual and variable absorption and emission centres. Thus, the primary goal to increase the concentration of co-dopants in UCNPs is to directly improve their brightness. However, the constraint of concentration quenching that limits the amount of the dopants usable has been known for years in bulk materials (for example, $\mathrm{Nd}^{3+}$-doped YAG laser crystals) $)^{12}$, and tuning the luminescence properties has been largely hindered at relatively low-doping concentrations ${ }^{2,13}$. For nanomaterials with a high ratio of surface area to volume, high-doping concentration is likely to induce both cross-relaxation energy loss and energy migration to the surface quenchers. This, in turn, explains the much

\footnotetext{
${ }^{1}$ Institute for Biomedical Materials \& Devices (IBMD), Faculty of Science, University of Technology Sydney, Sydney, NSW 2007, Australia. ${ }^{2}$ Department of Chemistry, National University of Singapore, 3 Science Drive 3, Singapore 117543, Singapore. ${ }^{3}$ Institute of Low Temperature and Structure Research, Polish Academy of Sciences, Okólna 2, 50-422 Wroclaw, Poland. ${ }^{4}$ Wroclaw Research Center, EIT+, Stablowicka 147, 54-066 Wroclaw, Poland. Correspondence and requests for materials should be addressed to A.B. (email: a.bednarkiewicz@int.pan.wroc.pl) or to X.L. (email: chmlx@nus.edu.sg) or to D.J. (email: dayong.jin@uts.edu.au)
} 
reduced luminescence quantum yield in upconversion nanomaterials relative to their bulk counterparts ${ }^{14-16}$. Encouragingly, over the past decade, a great deal of research efforts has been devoted to the study of the concentration quenching mechanisms $s^{2,10,17,18}$, thereby opening the door to many ground-breaking applications.

In this review, we discuss the phenomenon and underlying mechanism of concentration quenching occurring in UCNPs, review the general and emerging strategies for overcoming the concentration quenching effect, and summarize the impact of highly doped UCNPs on a range of disruptive applications. In particular, we discuss the rational design of heterogeneously doped, multilayered UCNPs that allow us to precisely control the energy migration process and induce cross-relaxation between the dopants for unprecedented optical phenomena. We present the challenges and opportunities of the doping strategies in developing smaller and brighter nanoparticles as well as hybrid materials with synergetic multifunction.

Concentration quenching. For a very long time, the problem of concentration quenching was the major obstacle that hindered the quest for highly luminescent materials ${ }^{12,19}$. The theory of concentration quenching in inorganic phosphors was introduced in 1954 by Dexter and Schulman, who pointed out that considerable quenching of luminescence in bulk materials arises when the activator concentration reaches $10^{-3}-10^{-2} \mathrm{M}[19]$. Different mechanisms (resonance energy transfer ${ }^{20}$, molecular interactions ${ }^{21}$, and intermolecular photo-induced electron transfer ${ }^{22}$ ) of concentration quenching in organic dyes have been studied since the early 1980s. The issue has limited the maximum number of fluorophores allowed in dye-doped silica nanoparticles ${ }^{23}$. As a result, the detrimental effect of concentration quenching in luminescent materials imposes a restriction on access to a high level of luminescence intensity, in consequence hindering their further applications.

The limitation set by the threshold of concentration quenching becomes a real problem for nanoscale luminescent materials (Figure 1a). As illustrated in Figure 1b, c, the general cause follows that high-doping concentration (shorter distance) leads to increased occurrence of energy transfer process between the dopants ${ }^{5,7}$. The excited-state electrons can be quickly short-circuited to the surface of nanomaterials, where a relatively large number of quenchers exist. Therefore, a dramatic decrease in luminescence intensity is observed. More specifically, the high-doping concentration facilitates both the energy migration of excited levels (typically within the sensitizersensitizer network) to the surface quenchers (Figure 1b) $15,24,25$ and the inter-dopant (typically between activators) crossrelaxation that causes emission intensity loss each time ${ }^{7,13}$ (Figure 1c).

To avoid the quenching of luminescence, conventionally, the doping level has been kept relatively low to ensure a sizable separation between the dopants to prevent parasitic interaction. Accordingly, the critical distance (Förster critical distance) is typically in the range of $2-6 \mathrm{~nm}^{26}$, meaning that the doping range should remain below $10^{-3} \mathrm{M}$ for organic dye-doped $\mathrm{SiO}_{2}{ }^{23}$ and $10^{-2} \mathrm{M}$ for UCNPs (Box 1) $)^{13}$. For an efficient upconversion to proceed, the relatively low concentrations of sensitizers (typically around $20 \mathrm{~mol} \%$ ) and activators (below $2 \mathrm{~mol} \%$ ) are generally used in the hexagonal-phase alkaline rare-earth fluoride nanocrystal, $\beta-\mathrm{AREF}_{4}$, that is known as one of the most efficient host material for upconversion. Low-doping concentration is the key roadblock to yield smaller and brighter luminescent nanomaterials ${ }^{27-29}$, which requires a canonical approach to optimizing the composition and chemical architecture of nanoparticles as well as photoexcitation schemes ${ }^{4,6}$.

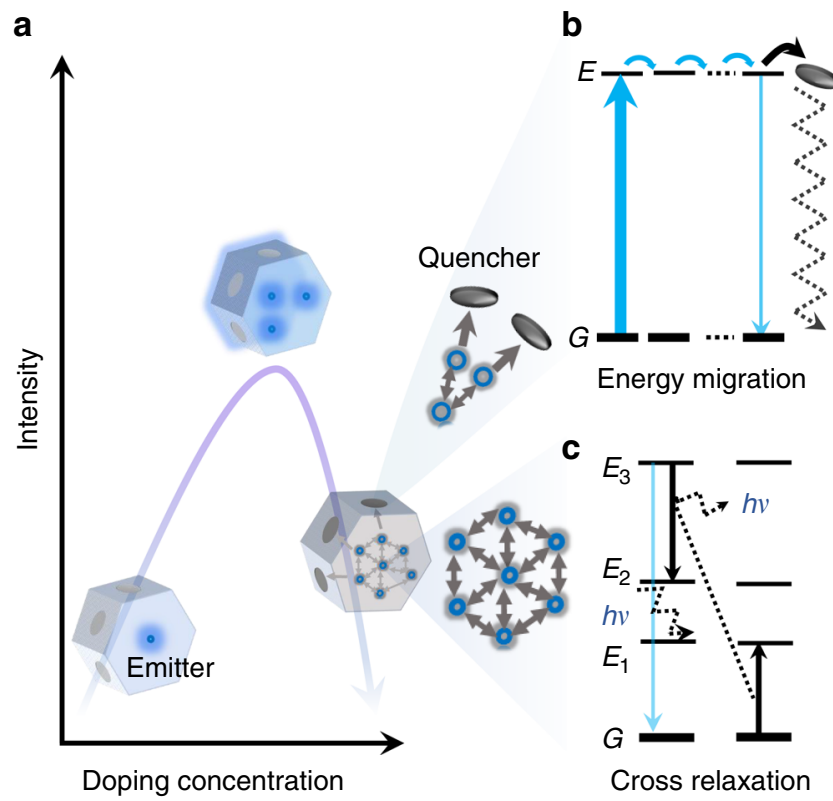

Fig. 1 Concentration quenching in upconversion nanoparticles. a Increasing the doping concentration of dopant ions in the nanoparticles increases the number of photon sensitizers and emitters, shortens the distance from sensitizer to activator, and hence enhances the emission brightness, but surpassing a concentration threshold could make the cascade energy transfer process less effective, as the concentration quenching dominates with high levels of dopants. In a highly doped system, the concentration quenching is likely to be induced by: $\mathbf{b}$ non-radiative energy migration to surface quenchers and c cross-relaxation non-radiative energy loss. The term $h v$ represents the phonon energy

Emerging strategies to overcome concentration quenching. Recently, great efforts have been made to overcome concentration quenching in luminescent nanoparticles, particularly from the upconversion research community. Various approaches have been developed to alleviate the threshold of concentration quenching in both homogeneously doped nanocrystals and heterogeneously doped core@shell nanocrystals. Box 2 summarizes four strategies that have proven effective for homogeneously doped nanocrystals. One of the most commonly used schemes is to passivate the particle's surface with an inert shell (for example, $\mathrm{NaYF}_{4}, \mathrm{NaGdF}_{4}$, $\mathrm{NaLuF}_{4}$ or $\mathrm{CaF}_{2}$ ), which can block the pathway of energy migration to surface quenchers ${ }^{15,25,36}$. The second strategy is to irradiate the particles with a high-energy flux that is sufficient for the activation of all dopant ions ${ }^{37}$. The third strategy is to choose a host crystal featuring a large unit cell to keep the D-A distance large enough even for stoichiometric compositions ${ }^{24,38}$. And the last strategy is to improve the doping uniformity in the host nanomaterials ${ }^{36,39}$, which minimizes the segregation of ions and thus prevents local concentration quenching.

Using wet-chemical synthesis methods developed over the past decade, it becomes possible to accurately control both the number and spatial distribution of dopants. This paves the way for a more efficient synthesis of heterogeneously doped core@multishell nanocrystals, which allows for the optimization of doping concentrations in each layer and selective isolation of different lanthanide ions to lower the probability of deleterious cross-relaxation. For example, Pilch et al. systematically studied a series of core@shell UCNPs to evaluate the effect of core@shell architecture on sensitizer and activator ions ${ }^{40}$; by separating sensitizers and emitters through the use of multilayer core@shell nanostructures, the concentration quenching threshold of $\mathrm{Er}^{3+}$ was lifted from 2 to $5 \%{ }^{41}$. 


\section{Box 1. | The mechanism of concentration quenching}

The notorious photophysical phenomenon of concentration quenching is frequently observed in solutions containing a high concentration of organic dyes, typically in the range of $10^{-3}-10^{-2} \mathrm{M}^{7,9,19,20}$. The leading factors for concentration quenching involve Förster resonance energy transfer (FRET) and Dexter electron transfer (DET). FRET is based on classical dipole-dipole interactions between the transition dipoles of the donor (D) and acceptor (A). The rate of the energy transfer decreases with the D-A distance, $R$, falling off at a rate of $1 / R^{6}$. DET is a short-range phenomenon that falls off exponentially with distance (proportional to $\mathrm{e}^{-k R}$, where $k$ is a constant that depends on the inverse of the van der Waals radius of the atom) and depends on spatial overlap of donor and quencher molecular orbitals ${ }^{16}$. The concentration quenching for phosphors is expressed by the rate constant, $k_{\mathrm{CQ}}$, determined from the equation for $\eta_{\mathrm{PL}}:{ }^{30}$

$$
\begin{gathered}
k=\frac{1}{T_{P L}}\left(\frac{R_{0}}{R}\right)^{6} \\
R_{0}=\sqrt[6]{\frac{9000 c^{4} \ln 10 x^{2} \eta_{P L}}{128 \pi^{5} n^{4} N_{A}} \int f_{D}(v) \varepsilon_{A}(v) \frac{d v}{v^{4}}} \\
\eta_{P L}=\eta_{e t} \eta_{i s c} \frac{k_{p h}}{k_{p h}+k_{n r}+k_{C Q}}
\end{gathered}
$$

where $T_{\mathrm{PL}}$ is the intrinsic radiative decay time of the $\mathrm{D}, R$ is the distance between $\mathrm{D}-\mathrm{A}$ and $R_{\mathrm{O}}$ is the Förster radius at which the energy transfer efficiency between $\mathrm{D}$ and $\mathrm{A}$ falls to $50 \%, \eta_{\mathrm{et}}$ is the energy transfer efficiency, $\eta_{\mathrm{isc}}$ is the intersystem crossing efficiency, $k_{\mathrm{ph}}$ and $k_{\mathrm{nr}}$ are the rate constants of radiative and non-radiative decay, respectively.

When this analogy extends to an inorganic system, such as UCNPs, the concentration quenching denotes the emission intensity decrease phenomenon as the dopant concentration is too high. Typically, the UCNPs contain two types of lanthanide dopants, that is, the sensitizer (D) and activator/emitter $(\mathrm{A})^{2,7}$. Though some singly doped (for example $\mathrm{Er}^{3+}$ ) particles can generate upconversion, researchers prefer to employ a sensitizer (for example $\mathrm{Yb}^{3+}$ ) to enlarge the absorbance in the NIR for enhanced upconversion luminescence. The process of the energy extraction from a sensitizer to an acceptor usually takes place via a non-radiative exchange (DET) or a multipolar interaction (FRET) ${ }^{31}$.

Most of the lanthanides have plentiful excited states, which show a high possibility to couple with one another through multipolar interactions with

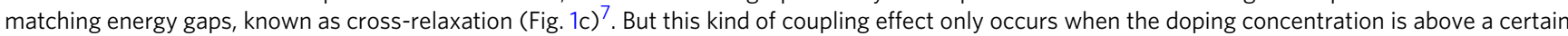
threshold. Because the $4 f-4 f$ transitions are symmetry forbidden according to the Laporte selection rule, the consequences are low intensity and longdecay time ${ }^{32,33}$. This cross-relaxation is primarily responsible for concentration quenching because neighbour ions, one in the excited state and the other in the ground state, non-radiatively exchange energy generally followed by phonon relaxation. The cross-relaxation process is evidenced by shorter lifetimes and decreased luminescence intensity.

Considering the energy transfer between two, or a network of, identical ions (for example, $\mathrm{Yb}^{3+}$ ions) in a concentrated system, the excited electrons will hop among the network. Such a process can quickly bring the excitation energy far away from the initial excited sensitizer, known as energy migration (Fig. 1b). In some specific systems, energy migration may be advantageous because it enables the upconversion from $\mathrm{Eu}^{3+} \mathrm{or} \mathrm{Tb}^{3+} \mathrm{emitters}^{3}$ infused with an $\mathrm{Gd}^{3+}$ ion network ${ }^{34}$, or for $\mathrm{Nd}^{3+}$-based sensitization with the assistance of an $\mathrm{Yb}^{3+}$ ion network ${ }^{35}$. However, these systems would short-circuit the excitation energy to quenchers.

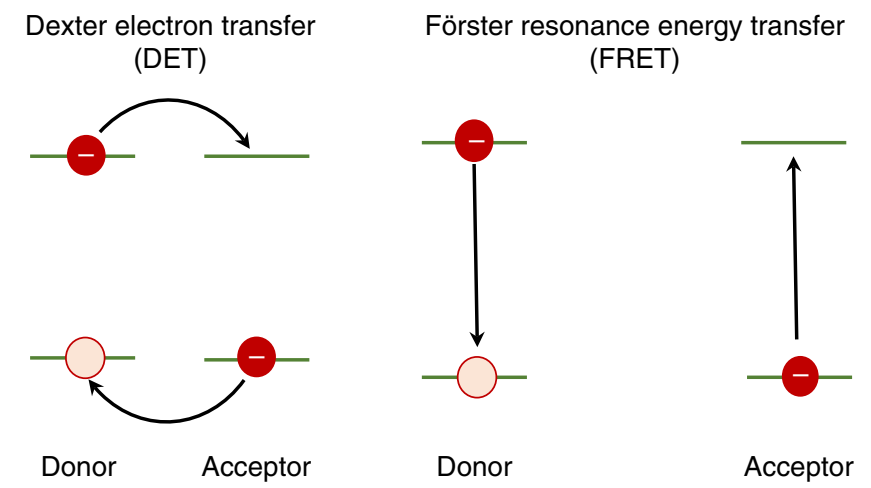

Homogeneously doped nanocrystals. Building brighter nanocrystals: Highly doped homogeneous UCNPs displaying exceptional brightness were first reported by Zhao and coworkers $^{17}$. As shown in Figure 2a, an increase in the excitation irradiance from $1.6 \times 10^{4}$ to $2.5 \times 10^{6} \mathrm{~W} \mathrm{~cm}^{-2}$ enhances the overall luminescence intensity by a factor of 5.6, 71 and 1105 for $0.5,4$ and $8 \% \mathrm{Tm}^{3+}$-doped nanocrystals, respectively ${ }^{17}$. The high brightness of these $\mathrm{Tm}^{3+}$-doped UCNPs allows the tracking of single nanoparticles in living cells through an optical microscope $e^{44}$. A similar trend was observed in highly $\mathrm{Er}^{3+}$-doped $\mathrm{NaYF}_{4}: \mathrm{Yb}^{3+}$ sub-10 nm nanocrystals (Figure 2b). As the excitation power increases, conventional UCNPs $\left(2 \% \mathrm{Er}^{3+}\right)$ saturate in brightness while the highly doped UCNPs $\left(20 \% \mathrm{Er}^{3+}\right)$ appear much brighter than the conventional $\mathrm{UCNPs}^{18}$. The optimal concentration for $\mathrm{Nd}^{3+}$ (conventionally around 1\%) was increased to $20 \%$ with the sensitization of indocyanine green (ICG), resulting in about a tenfold brightness enhancement (Figure 2c $)^{45}$. Encouragingly, the upconversion emission from a series of $\mathrm{NaYF}_{4}: x \% \mathrm{Er}^{3+} @ \mathrm{NaLuF}_{4}$ nanocrystals clearly showed high brightness at high-doping concentrations of $\mathrm{Er}^{3+}$, with $\mathrm{NaErF}_{4} @ \mathrm{NaLuF}_{4}(100 \%$ doping) being the brightest (Fig. 2d) ${ }^{25}$. 


\section{Box 2. I Main strategies to overcome concentration quenching}

a Coating an inert shell: Inert shell passivation on a low-doping luminescent core is a common strategy to avoid surface quenching by shielding the luminescent core from surface quenchers. This strategy further helps highly doped UCNPs by preventing the migration of sensitized photon energy to the surface quenchers, providing a convenient solution for detrimental quenching effects ${ }^{15,25,36,42}$.

b Increasing excitation power density: In the case of low irradiance, concentration quenching happens in highly doped UCNPs because too many nearby ground-state ions will easily take away the energy of excited-state ions. By supplying a high irradiance, either by using a high-power laser or focusing the excitation beam, a sufficient amount of excitation photon flux will be supplied to the large number of highly doped ions, and the majority of them will be at excited (intermediate) states, which reduces the number of detrimental ground-state ions. This strategy has proven highly effective in alleviating the thresholds of concentration quenching in UCNPs involving $\mathrm{Tm}^{3+} \mathrm{or} \mathrm{Er}^{3+}$ as emitters ${ }^{17,18}$. Benz et al. also described a rate equation model, which shows that the increased luminescence intensity for highly doped nanocrystals at a high irradiance is due to the enlarged distance between excited ions and ground-state ions ${ }^{37}$.

c Choosing a host nanocrystal with a large unit cell size 24,38 : The minimum distance between two dopants in a nanocrystal is determined by the size of the unit cell. Taking the $\beta-\mathrm{NaYF}_{4}$ crystalline structure as an example, the average distance between a sensitizer and an acceptor can be approximated using the following equation: $d_{\beta-\mathrm{NaYF}_{4}}=\left(\frac{a^{2} c \sqrt{3} / 2}{1.5(x+y)}\right)^{1 / 3}$, which is evolved from the known lattice parameters when ignoring the lattice distortion caused by doping ${ }^{31}$. The parameters $x$ and $y$ represent the doping concentrations of the sensitizer and acceptor, respectively, while $a$ and $c$ are the lattice parameters of the hexagonal unit cells.

d Improving the homogeneous distribution of dopants: A homogeneous distribution of dopants can avoid local concentration quenching. The layer-bylayer hot injection strategy is technically superior to conventional one-pot heating up synthesis procedures in achieving a high uniformity in the dopant distribution ${ }^{43}$. Precise control on the Angstrom scale of the dopant distribution may be achieved by managing the rate of precursor injection ${ }^{43}$.

a
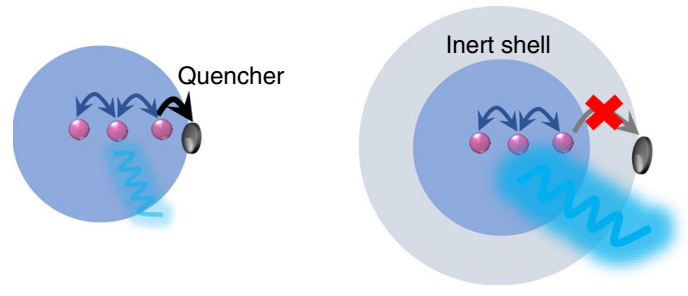

C
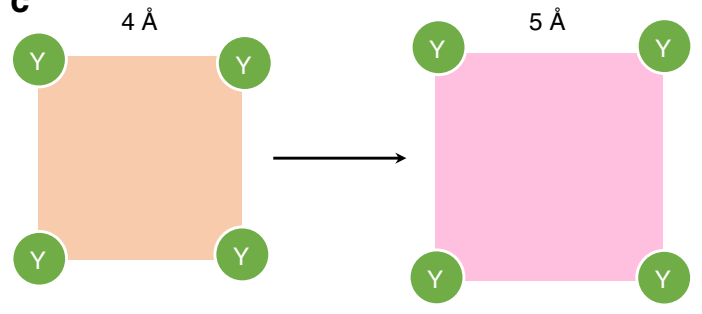

b

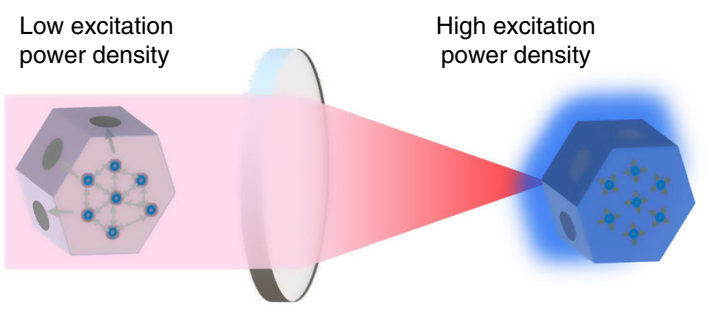

d

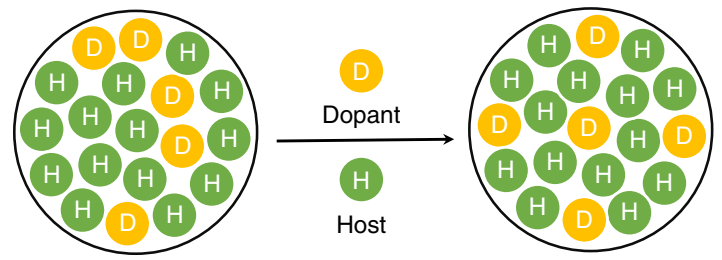

The optimal concentration of $\mathrm{Yb}^{3+}$ sensitizer ions has also been pushed to its limit. Chen et al. reported that highly $\mathrm{Yb}^{3+}$-doped sub-10 nm cubic phase $(a-) \mathrm{NaYF}_{4}: \mathrm{Yb}^{3+}, \mathrm{Tm}^{3+}$ nanocrystals display a 43 times emission enhancement at NIR wavelengths compared to the conventional $20 \% \mathrm{Yb}^{3+}$-doped one ${ }^{46}$. With an inert shell coating, the $\alpha-\mathrm{NaYbF}_{4}: 2 \% \mathrm{Er}^{3+} @ \mathrm{CaF}_{2} \mathrm{UCNPs}$ display a 15 -fold enhancement in visible light emission (Figure 2e) 47. Using an inert $\mathrm{CaF}_{2}$ shell, heavy doping of $\mathrm{Yb}^{3+}$ ions in the ultra-small $a-\mathrm{NaYF}_{4}: \mathrm{Yb}^{3+}, \mathrm{Tm}^{3+}$ and $\alpha-\mathrm{NaYbF}_{4}: \mathrm{Tb}^{3+}$ nanoparticles was found to dramatically enhance the upconversion emission for high-contrast bioimaging ${ }^{48-51}$. With a $\mathrm{NaYF}_{4}$ inert shell, the optimal concentration of $\mathrm{Yb}^{3+}$ sensitizer ions was quantitatively examined by characterizing $\beta$-phase UCNPs at the single-particle level ${ }^{52}$.

Emerging applications: With exceptional brightness and associated optical properties, a range of applications have emerged. For example, highly $\mathrm{Tm}^{3+}$-doped UCNPs enable single nanoparticle sensitivity using a suspended-core microstructured optical-fibre dip sensor (Fig. 2f) ${ }^{17,53}$. The diverging brightness trends enable the optical encoding application by varying the excitation intensities ${ }^{18}$. More recently, $\mathrm{Li}$ et al. employed living yeast as a natural biomicrolens to concentrate the excitation energy of light and to enhance upconversion luminescence, suggesting a new way of detection of cells ${ }^{54}$.

High efficiency of upconversion emission from the NIR to the $\mathrm{UV}$ was achieved by high $\mathrm{Yb}^{3+}$ doping in $\mathrm{NaGdF}_{4}: 70 \% \mathrm{Yb}^{3+}, 1 \%$ $\mathrm{Tm}^{3+} @ \mathrm{NaGdF}_{4}$ nanostructures, and by varying the NIR excitation power density the reversible dynamic red, green and blue reflections of superstructure in a single thin film was demonstrated (Figure $2 \mathrm{~g})^{55}$. Through confined energy migration, an efficient fivephoton upconverted UV emission of $\mathrm{Tm}^{3+}$ has been demonstrated in a $\mathrm{NaYbF}_{4}$ host without concentration quenching, and the large amount of spontaneous upconversion emission provides sufficient gain in a micron-sized cavity to generate stimulated lasing emissions in deep-ultraviolet (around $311 \mathrm{~nm}$ ) wavelength (Figure $2 \mathrm{~h})^{56}$.

Using an increased excitation power density and highly $\mathrm{Er}^{3+}$. doped $\mathrm{NaYF}_{4}: 20 \% \mathrm{Yb}^{3+}, 20 \% \mathrm{Er}^{3+} @ \mathrm{NaYF}_{4}$ UCNP, Drees et al. reported that resonance energy transfer has been enhanced by more than two orders of magnitude compared to that of standard $\mathrm{NaYF}_{4}: 20 \% \mathrm{Yb}^{3+}, 2 \% \mathrm{Er}^{3+} @ \mathrm{NaYF}_{4}$ particles being excited at a lowpower density ${ }^{57}$. After conjugation with anti-GFP nanobodies, the formed UCNP nanoprobe was used to target GFP fusion proteins inside living cells via a blue upconversion luminescence- 
a

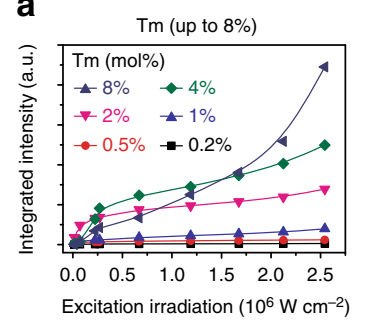

b

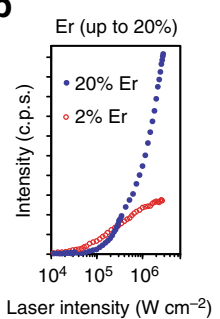

C

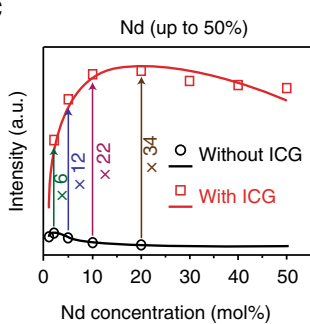

d

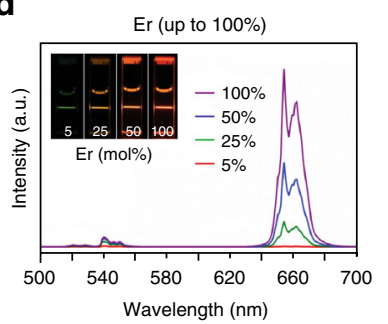

e

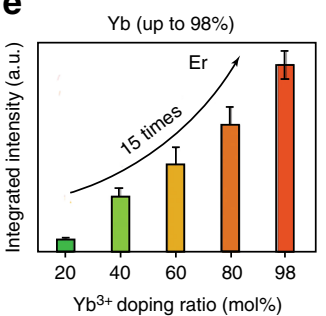

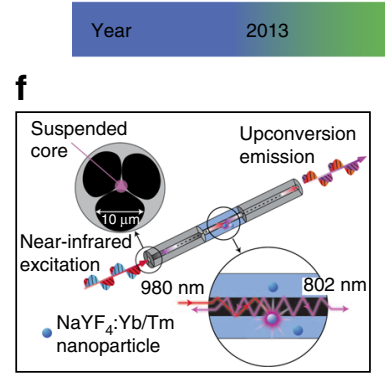

Single nanocrystal tracking

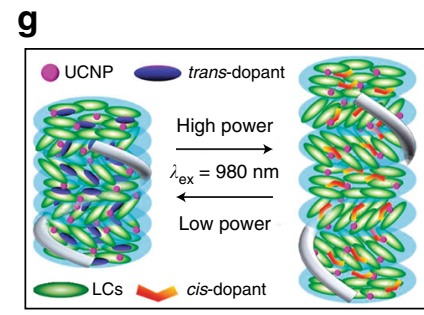

NIR-triggered reflection

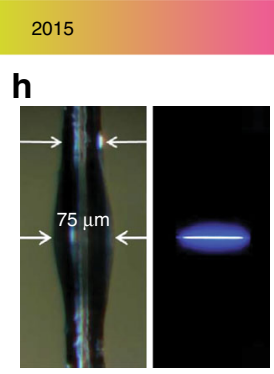

Upconversion lasing

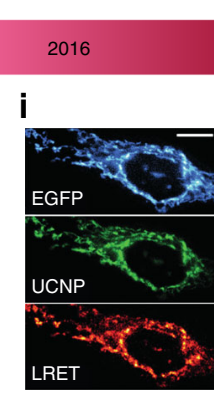

Protein interactions

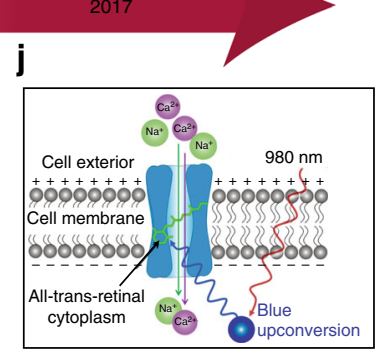

Subcellular optogenetics

Fig. 2 Selected milestones overcoming concentration quenching in homogeneously doped upconversion nanocrystals. a Integrated upconversion luminescence intensity as a function of excitation irradiance for a series of $\mathrm{Tm}^{3+}$-doped (0.2-8\%) nanocrystals. Adapted from ref. ${ }^{17}$. b Upconversion luminescence intensity of single $8 \mathrm{~nm}$ UCNPs with 20 and $2 \% \mathrm{Er}^{3+}$, each with $20 \% \mathrm{Yb}^{3+}$, plotted as a function of excitation intensity. Adapted from ref. ${ }^{18}$. c Experimental results (black circle and red square) and theoretical modelling (black and red curves) of integrated upconversion luminescence intensities of a set of $\mathrm{NaYF}_{4}: \mathrm{Nd}^{3}+$ UCNPs with and without indocyanine green (ICG) sensitization. Adapted with permission from ref. ${ }^{45}$ Copyright (2016) American Chemical Society. d Luminescence spectra of colloidal dispersion of NaYF $4: x \% E r @ N a L u F_{4}$ nanocrystals ( $\left.x=5,25,50,100\right)$; Inset: luminescence images of $\mathrm{NaYF}_{4}: x \% \mathrm{Er}_{\mathrm{NNaLuF}}$ in cyclohexane excited with a $980 \mathrm{~nm}$ laser. Adapted with permission from ref. ${ }^{25}$ Copyright (2017) American Chemical Society. e Integrated upconversion luminescence intensity of $\alpha-\mathrm{NaY}_{0.98-x} \mathrm{Yb}_{x} \mathrm{~F}_{4}: 2 \% \mathrm{Er} @ \mathrm{CaF}_{2}(x=0.2,0.4,0.6,0.8,0.98)$. Adapted with permission from ref. 47 Copyright (2017) Royal Society of Chemistry. $\mathbf{f}$ Schematic of the experimental configuration for capturing upconversion luminescence of NaYF 4 :Yb ${ }^{3+}$, Tm $^{3+}$ nanocrystals using a suspended-core microstructured optical-fibre dip sensor. Adapted from ref. ${ }^{53}$. $\mathbf{g}$ Upon irradiation by a NIR laser at the high-power density, the reflection wavelength of the photonic superstructure red-shifted, whereas its reverse process occurs upon irradiation by the same laser but with the lower-power density. Adapted with permission from ref. ${ }^{55}$ Copyright (2014) American Chemical Society. $\mathbf{h}$ Photographs of a microresonator with and without optical excitation. Adapted from ref. ${ }^{56}$. i UCNPs functionalized with a nanobody recognizing enhanced green fluorescent protein (EGFP) could rapidly and specifically target to EGFP-tagged fusion proteins in the mitochondrial outer membrane, and this protein interaction process could be detected by lanthanide resonance energy transfer (LRET) in living cells. Scale bar: $10 \mu \mathrm{m}$. Reproduced with permission from Drees et al. ${ }^{57}$ copyright John Wiley and Sons. j Schematic of channelrhodopsin-2 activated in HeLa cells by strong blue upconversion luminescence from NaYbF 4 :Tm+@NaYF4 core@shell structure. Adapted with permission from ref. ${ }^{58}$ Copyright (2017) American Chemical Society

induced resonance energy transfer process (Figure $2 \mathrm{i})^{57}$. More recently, Pliss et al. reported that $\mathrm{NaYbF}_{4}: 0.5 \% \mathrm{Tm}^{3+} @ \mathrm{NaYF}_{4}$ UCNPs emit six times higher blue emission, compared to typical $\mathrm{NaYF}_{4}: 30 \% \mathrm{Yb}^{3+}, 0.5 \% \mathrm{Tm}^{3+} @ \mathrm{NaYF}_{4} \mathrm{UCNPs}$, for effective optogenetic activation using NIR light (Figure $2 \mathrm{j})^{58}$.

Heterogeneously doped nanocrystals. The precision in controlled growth has resulted in a library of intentional heterogeneously doped core@shell UCNPs ${ }^{59,60}$. The doping concentrations in multilayers of nanostructure can be optimized to satisfy the requirements of a particular application, for example, according to either excitation conditions ${ }^{16,35,61,62}$ and/ or desirable emission wavelengths ${ }^{34,63,64}$, to produce a highperformance energy-migration-mediated upconversion process ${\text { (Figure } 3)^{16} \text { or efficient light-to-heat conversion }}^{65}$.

Controlled energy migration: As shown in Figure 3a, the finetuning of upconversion emission colours through energy migration has been first demonstrated using a $\mathrm{Gd}^{3+}$ sublattice structure as an efficient energy transfer bridge across the core@shell interface. As shown in Figure 3b, upconversion emission with tunable wavelengths and lifetimes has been realized via a prescribed energy cascade of $\mathrm{Yb}^{3+} \rightarrow \mathrm{Tm}^{3+} \rightarrow \mathrm{Gd}^{3+} \rightarrow$ lanthanide activators (for example, $\mathrm{Tb}^{3+}, \mathrm{Eu}^{3+}, \mathrm{Dy}^{3+}$ and $\left.\mathrm{Sm}^{3+}\right)^{34}$. Note that the efficiency of energy-migration-mediated upconversion emission of $\mathrm{Tb}^{3+}, \mathrm{Eu}^{3+}$ or $\mathrm{Dy}^{3+}$ at high-doping concentrations is more than two orders of magnitude of that from a $\mathrm{Yb}^{3+}$-sensitized cooperative energy transfer system ${ }^{32}$. Efficient photon upconversion has also been demonstrated through the heterogeneous core@shell nanostructure of $\mathrm{NaYbF}_{4}: \mathrm{Gd}^{3+}, \mathrm{Tm}^{3+} @ \mathrm{NaGdF}_{4} @ \mathrm{CaF}_{2}: \mathrm{Ce}^{3+}$ with a high-doping concentration of $\mathrm{Ce}^{3+}$ in the shell layer ${ }^{66} \cdot \mathrm{A} \mathrm{CaF}_{2}$ host has been employed to reduce the $4 f-5 d$ excitation frequency of $\mathrm{Ce}^{3+}$ to match the energy level of $\mathrm{Gd}^{3+}$. Zhang et al. have fabricated $\mathrm{NaGdF}_{4}: \mathrm{Yb}^{3+}$, $\mathrm{Tm}^{3+}, \mathrm{Er}^{3+} @ \mathrm{NaGdF}_{4}: \mathrm{Eu}^{3+} @ \mathrm{NaYF}_{4}$ incorporated with RGBemitting lanthanide ions at high concentration, and generated high-brightness white light across the whole visible spectrum ${ }^{67}$. Notably, apart from the lanthanide ions, organic dyes tethered on the surface of $\mathrm{NaGdF}_{4}: \mathrm{Yb}^{3+}, \mathrm{Tm}^{3+} @ \mathrm{NaGdF}_{4}$ nanocrystals can accept the sensitized UV energy through the $\mathrm{Gd}^{3+}$-mediated energy transfer, which can dramatically improve the sensitivity in FRETlimited measurements ${ }^{68}$. Apart from $\mathrm{Gd}^{3+}$ as the energy mediation ions, due to a large energy gap $(\Delta E>5 h v)$ between respective levels ${ }^{69,70}, \mathrm{Mn}^{2+}$ and $\mathrm{Tb}^{3+}$ ions also show a similar function for energy migration from the core to shell when doped by high concentration of activators.

Exciting UCNPs at $980 \mathrm{~nm}$, through the transparent biological window (650-1350 nm), offers higher photo-biocompatibility and allows deeper tissue penetration than that achievable at $532 \mathrm{~nm}$, as living cells can withstand around 3000 times more intensity at 980 
a

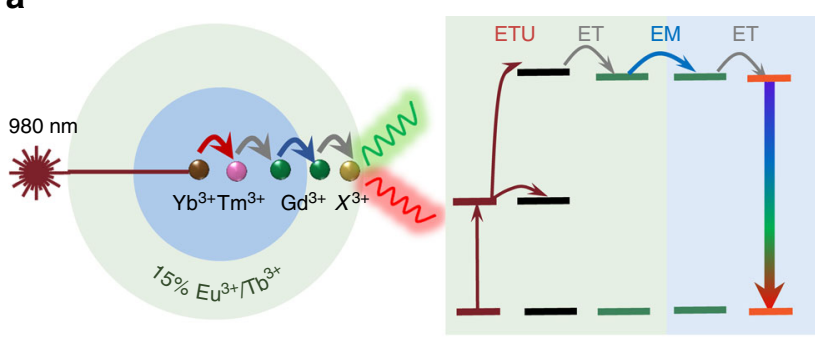

C

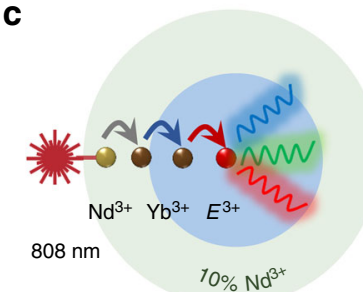

e
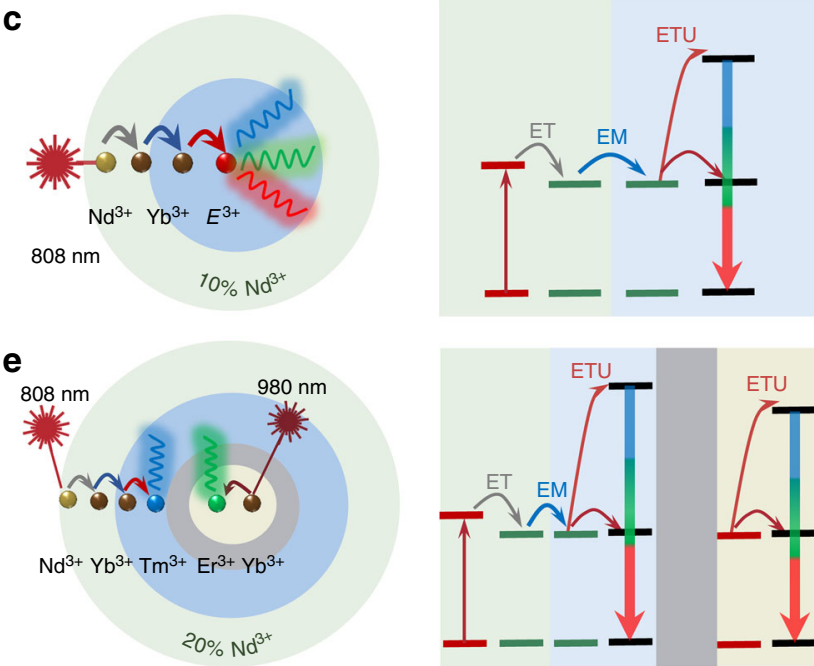

b
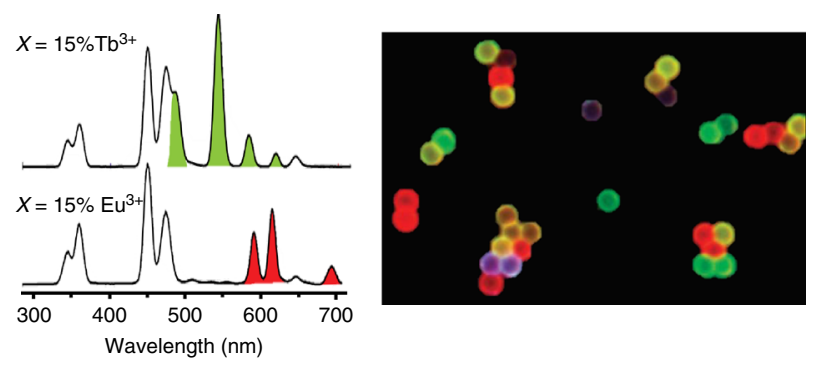

d

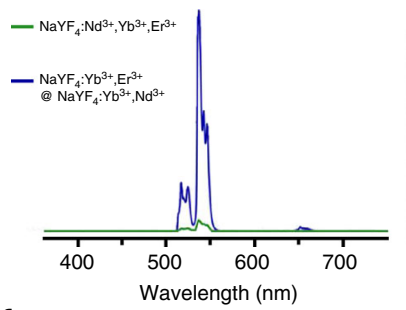

$808 \mathrm{~nm}$ excitation $\quad 980 \mathrm{~nm}$ excitation

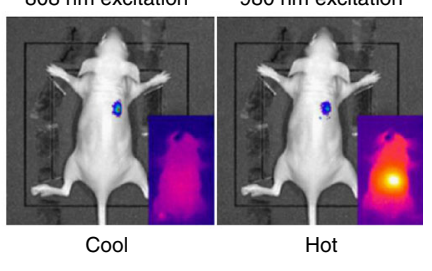

f
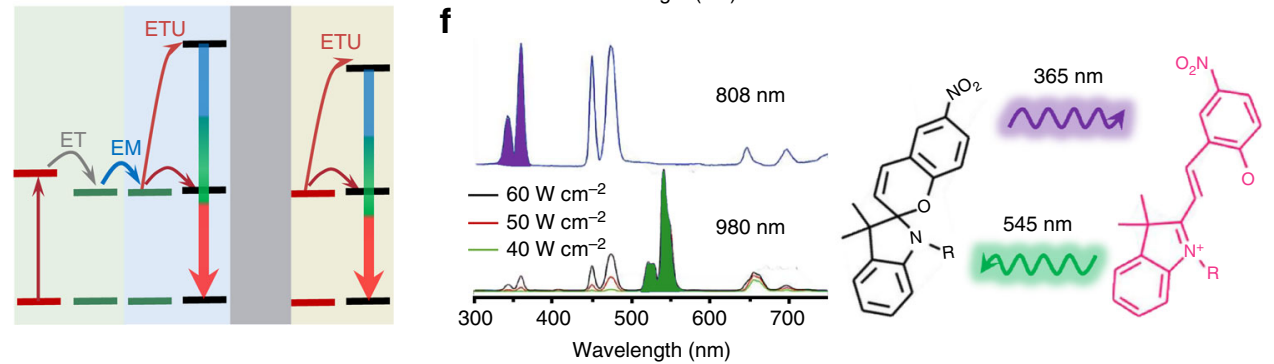

Fig. 3 Heterogeneously doped core@shell upconversion nanoparticles to overcome concentration quenching. a The schematic of a core@shell design with energy migration at the emission part ${ }^{34}$. b Upconversion emission spectra with tunable wavelengths attributed to a prescribed $\mathrm{Yb}^{3+} \rightarrow \mathrm{Tm}^{3+} \rightarrow \mathrm{Gd}^{3+} \rightarrow$ $\mathrm{Eu}^{3+} / \mathrm{Tb}^{3+}$ energy cascade across the core@shell interface, and luminescence micrograph of polystyrene beads tagged with core@shell nanoparticles. Adapted from ref. ${ }^{34}$. c Schematic of the core@shell design with energy migration at the excitation part, $\mathrm{E}^{3+}=\mathrm{Er}^{3+}, \mathrm{Tm}^{3+}$, or $\mathrm{Ho}^{3+} 35$. d Upconversion emission spectra of $\mathrm{NaYF}_{4}: \mathrm{Yb}^{3+}, \mathrm{Er}^{3+} @ \mathrm{NaYF}_{4}: \mathrm{Yb}^{3+}{ }_{,} \mathrm{Nd}^{3+}$ core@shell and homogeneous-doped nanoparticles, and the in vivo imaging of the core@shell nanoparticles under $808 \mathrm{~nm}$ and $980 \mathrm{~nm}$ excitation, respectively. Adapted with permission from ref. ${ }^{35}$ Copyright (2013) American Chemical Society. e Schematic of doping location control in the core@shell design with an energy transfer blocking layer ${ }^{80}$. $f$ Core@multishell UCNPs $\left(\mathrm{NaYF}_{4}: \mathrm{Yb}^{3+}{ }^{3}, \mathrm{Nd}^{3+}{ }^{3} \mathrm{Tm}^{3}\right.$ ${ }^{+} @ \mathrm{NaYF}_{4} @ \mathrm{NaYF}_{4}: \mathrm{Yb}^{3+}{ }_{,} \mathrm{Er}^{3+}$ ) with orthogonal emissions under irradiation at 800 and $980 \mathrm{~nm}$, and the orthogonal emissions were employed for reversible isomerization of spiropyran derivatives. Adapted with permissions from Lai et al. ${ }^{80}$ Copyright John Wiley and Sons

$\mathrm{nm}$ than at $532 \mathrm{~nm}$ visible excitation ${ }^{71}$. Considering the fact that water absorbs around 20 times more excitation light at $980 \mathrm{~nm}$ than at $800 \mathrm{~nm}$, researchers have further designed core@shell UCNPs by shifting the excitation wavelength to around $800 \mathrm{~nm}^{35}$. The key is to use $\mathrm{Nd}^{3+}$ ions as the sensitizer to absorb $800 \mathrm{~nm}$ photons. Because the absorption cross-section of $\mathrm{Nd}^{3+}$ ions at $800 \mathrm{~nm}$ is 25 -fold larger than that of $\mathrm{Yb}^{3+}$ ions at $980 \mathrm{~nm}, \mathrm{Nd}^{3+}$-doped UCNPs display brighter upconversion emissions and negligible overheating effects $^{72}$. Since homogeneously co-doping high concentration of $\mathrm{Nd}^{3+}$ ions and activators will quench the overall upconversion emission, owing to the deleterious back energy transfer from activators to $\mathrm{Nd}^{3+}$ ions, the doping concentration of $\mathrm{Nd}^{3+}$ has been limited to below $1 \%$. To overcome this threshold, an energy migration system has been employed to separate $\mathrm{Nd}^{3+}$ ions and activators (Figure 3c). Yan et al. first demonstrated highconcentration doping of $\mathrm{Nd}^{3+}$ in core@shell UCNPs that displayed a much enhanced upconversion luminescence relative to those homogeneously doped (Figure $3 \mathrm{~d}$ ). Using these $\mathrm{Nd}^{3+}$-sensitized UCNPs, the authors further demonstrated superior imaging performance for in vivo imaging without the issue of tissue overheating (Figure $3 \mathrm{~d})^{35}$. However, further studies are still needed to investigate the penetration depth trade-off of using $800 \mathrm{~nm}$ excitation light, since the amount of light scattering increases in proportion to the fourth power of the frequency of the light. To this regard, a future research direction is to shift the excitation wavelength from the first NIR optical window $(650-1000 \mathrm{~nm})$ to the second NIR spectral window (1100-1350 nm), which is ideal for deep-tissue imaging because of reduced water absorption and light scattering $^{73}$.

To optimize the doping concentration in core@shell structures, $\mathrm{Xie}$ et al. have found that the design of $\mathrm{NaYF}_{4}: 20 \% \mathrm{Yb}^{3+}, 0.5 \%$ $\mathrm{Tm}^{3+}, 1 \% \mathrm{Nd}^{3+} @ \mathrm{NaYF}_{4}: 20 \% \mathrm{Nd}^{3+}$ results in upconversion emission around seven times stronger than that of $\mathrm{NaYF}_{4}: \mathrm{Nd}^{3+}, \mathrm{Yb}^{3+}$, $\mathrm{Tm}^{3+} @ \mathrm{NaYF}_{4}{ }^{61}$. To further reduce the cross-relaxation and back energy transfer from activators to $\mathrm{Nd}^{3+}$ sensitizers, Zhong et al. reported a nanostructure design in the form of $\mathrm{NaYF}_{4}: \mathrm{Yb}^{3+}$, $\mathrm{Er}^{3+} @ \mathrm{NaYF}_{4}: \mathrm{Yb}^{3+} @ \mathrm{NaNdF}_{4}: \mathrm{Yb}^{3+}$, in which the intermediate $\mathrm{NaYF}_{4}: \mathrm{Yb}^{3+}$ shell separates $\mathrm{Er}^{3+}$ activators from $\mathrm{Nd}^{3+}$ primary sensitizers ${ }^{74}$. With the doping concentration of $\mathrm{Nd}^{3+}$ being pushed to $90 \%$, there is eight times more upconversion luminescence produced, compared to the $\mathrm{NaYF}_{4}: \mathrm{Yb}^{3+}, \mathrm{Er}^{3+} @ \mathrm{NaYF}_{4}: \mathrm{Nd}^{3+}$ structure. Encouragingly, a more sophisticated doping pattern of $\mathrm{NaYF}_{4}: 2 \% \mathrm{Er}^{3+}, 30 \% \mathrm{Yb}^{3+} @ \mathrm{NaYF}_{4}: 20 \% \mathrm{Yb}^{3+} @ \mathrm{NaNdF}_{4}: 10 \% \mathrm{Yb}^{3+}$, with fine-tuning of $\mathrm{Yb}^{3+}$ concentrations at different layers, was found to facilitate more efficient energy transfer process of $\left(\mathrm{Nd}^{3+} \rightarrow\right.$ $\left.\mathrm{Yb}^{3+}\right) \rightarrow\left(\mathrm{Yb}^{3+}\right) \rightarrow\left(\mathrm{Yb}^{3+} \rightarrow \mathrm{Er}^{3+}\right)$ at higher $\mathrm{Nd}^{3+}$ doping concentration. This design further reduces the requirement in the excitation power, so that upconversion luminescence has been 
observed even under a $740 \mathrm{~nm} \mathrm{LED}{ }^{75}$. A similar energy migration strategy has been proposed to get cooperative emission from $\mathrm{Tb}^{3+}$ ions, which demonstrated a tenfold upconversion enhancement under $800 \mathrm{~nm}$ photoexcitation of $\mathrm{Nd}^{3+}$ ions as compared to $\mathrm{Yb}^{3+}$ sensitization. ${ }^{76}$ The ratiometric $\mathrm{Nd}^{3+} \rightarrow \mathrm{Yb}^{3+}$ and $\mathrm{Yb}^{3+} \rightarrow \mathrm{Er}^{3+}$ energy transfer processes in the core@shell nanocrystals have also been used for temperature sensing in two different temperature ranges ${ }^{77}$. More detailed and systematic studies of the concentrations of both the primary sensitizer and secondary sensitizers will improve the efficiency of energy cascade.

Combining strategies presented above (Figure 3a, c), upconversion tuning with high-doping concentrations can be achieved by either $\mathrm{Gd}^{3+}$-mediated energy migration or $\mathrm{Yb}^{3+}$-mediated absorption/migration. For example, the core@multishell structure of $\mathrm{NaYbF}_{4}: 50 \% \mathrm{Nd}^{3+} @ \mathrm{NaGdF}_{4}: \mathrm{Yb}^{3+}, \mathrm{Tm}^{3+} @ \mathrm{NaGdF}_{4}: A$ ( $A$ : activator $\mathrm{Eu}^{3+}, \mathrm{Tb}^{3+}$, or $\mathrm{Dy}^{3+}$ ) has been utilized to initiate the energy migration from $\mathrm{Gd}^{3+}$ to the activators with high concentration ${ }^{78}$. In this system, the $\mathrm{Nd}^{3+}$-sensitized UCNPs displayed emissions spanning from the UV to the visible region with high efficiency through a single wavelength excitation at 808 $\mathrm{nm}$. More recently, Liu et al. designed a multilayer nanoparticle for simultaneously displaying short- and long-lived upconversion emission with high concentrations of different dopants at different layers, making multilevel anti-counterfeiting possible at a single-particle level ${ }^{79}$.

Blocking layer separation: In addition to the strategies of tuning excitation and emission properties, the control over doping location with a blocking layer (Figure 3e) has been demonstrated for orthogonal emissions ${ }^{78,80,81}$, spectral/lifetime multiplexing ${ }^{82}$, upconverting/downshifting ${ }^{83}$, multimode imaging ${ }^{84}$, and multi-optical functions in single particles ${ }^{65}$. For example, reversible isomerization of spiropyran derivatives has been achieved by the orthogonal emissions of core@multishell UCNPs with a high-doping concentration of $\mathrm{Nd}^{3+}$ under irradiation at 800 and $980 \mathrm{~nm}$ (Figure $3 \mathrm{f})^{80}$. A similar idea was used to efficiently trigger a reversible photocyclization of the chiral diarylethene molecular switch by the UV and visible luminescence from core@multishell UCNPs with dual wavelength NIR light transduction properties ${ }^{85}$. The emission colours of these $\mathrm{Ho}^{3+} / \mathrm{Tm}^{3+}$ co-doped $\mathrm{NaGdF}_{4}: \mathrm{Yb}^{3+} \mathrm{UCNPs}$ can be tuned by changing the laser power density or temperature, due to the different spectral responses ${ }^{86}$. By design and synthesis of $\mathrm{NaGdF}_{4}: \mathrm{Nd}^{3+} @ \mathrm{NaYF}_{4} @ \mathrm{NaGdF}_{4}: \mathrm{Nd}^{3}$ ${ }^{+}, \mathrm{Yb}^{3+}, \mathrm{Er}^{3+} @ \mathrm{NaYF}_{4}$ nanoparticles, both upconversion and downshifting luminescence, sensitized by highly doped $\mathrm{Nd}^{3+}$, can be achieved without cross interference ${ }^{83}$. Moreover, excited $\mathrm{Nd}^{3+}$ ions can transfer energy to other lanthanide ions and result in tunable downshifting emission. For instance, co-doping $\mathrm{Yb}^{3+}$ with $\mathrm{Nd}^{3+}$ at high concentrations would give an intense NIR emission centred around $980 \mathrm{~nm}$ due to the efficient energy transfer from $\mathrm{Nd}^{3+}$ to $\mathrm{Yb}^{3+87}$. The spectral and lifetime characteristics can correlate orthogonally with excitation by constructing noninterfering luminescent regions in a nanoparticle, which enables the multiplexed fingerprint and time-gated luminescent imaging in both spectral and lifetime dimensions ${ }^{82}$. More recently, Marciniak et al. have demonstrated the heterogeneous doping of $\mathrm{Nd}^{3+}$ ions with different concentrations in different parts of $\mathrm{NaNdF}_{4} @$ $\mathrm{NaYF}_{4} @ \mathrm{NaYF}_{4}: 1 \% \mathrm{Nd}^{3+}$ nanoparticles to achieve three optical functions, namely efficient $(\eta>72 \%)$ light-to-heat conversion, bright NIR emission and relatively sensitive $\left(S_{\mathrm{R}}>0.1 \% \mathrm{~K}^{-1}\right)$ localized temperature quantification ${ }^{65}$. The undoped $\mathrm{NaYF}_{4}$ intermediate shell enables the separation of the $1 \% \mathrm{Nd}^{3}$ ${ }^{+}$-doped outer shell (for efficient Stokes emission) from $100 \% \mathrm{Nd}^{3+}$-doped core (for cross-relaxation based efficient light-to-heat conversion).
Emerging applications enabled by cross-relaxation. Crossrelaxation has often been perceived as being deleterious, but new research shows that cross-relaxation can render many unique properties, such as single-band emission ${ }^{88,89}$, energy looping ${ }^{73}$, tunable colour/lifetime ${ }^{63,90}$, enhanced downshifting emissions ${ }^{91}$ and photo-avalanche effect for amplified-stimulated emission $^{1,92}$.

Single-band emission: High-throughput molecular profiling requires optical multiplexing of single-band emission probes to target multiple analytes without crosstalk (Figure $4 \mathrm{~b}$ ), but each lanthanide ion emitter in an UCNP has multiple energy levels with multiple emission peaks ${ }^{93}$. Cross-relaxation by high-doping concentration has been used to quench the unwanted emission bands to yield single-band emission ${ }^{94,95}$. Chan et al. used combinatorial screening of multiple doped $\mathrm{NaYF}_{4}$ nanocrystals to identify a series of doubly and triply doped nanoparticles with pure emission spectra at various visible wavelengths ${ }^{96}$. Approaching $100 \%$ red emission output has been reported by Wei et al. using highly doped activators, where the cross-relaxation effect dominates and quenches the green or blue emissions (Figure $4 a)^{89}$. This strategy was successful in achieving pure red 696 or $660 \mathrm{~nm}$ upconversion emission as well as precisely tuning upconversion colours to study the underlying upconversion mechanisms.

More recently, Chen et al. presented a new class of $\beta$ $\mathrm{NaErF}_{4}: 0.5 \% \mathrm{Tm}^{3+} @ \mathrm{NaYF}_{4}$ nanocrystals with bright red upconversion luminescence through high concentration $\mathrm{Er}^{3+}$-based host sensitization, in which $\mathrm{Tm}^{3+}$ ions are employed to trap excitation energy and to minimize the luminescence quenching effect ${ }^{97}$. Introducing high concentrations of $\mathrm{Ce}^{3+}$ into $\mathrm{NaYF}_{4}: \mathrm{Yb}^{3+} / \mathrm{Ho}^{3+}$ or $\mathrm{NaYF}_{4}: 40 \% \mathrm{Gd}^{3+}$ have greatly enhanced the red-to-green upconversion emission ratio of $\mathrm{Ho}^{3+}$ through effective crossrelaxation between $\mathrm{Ce}^{3+}$ and $\mathrm{Ho}^{3+98,99}$. Similarly, combining the strategy presented in Figure $3 \mathrm{c}$, single-band red upconversion luminescence of $\mathrm{Ho}^{3+}$ has been achieved under $808 \mathrm{~nm}$ excitation from $\mathrm{NaGdF}_{4}: \mathrm{Yb}^{3+}{ }_{,} \mathrm{Ho}^{3+}, \mathrm{Ce}^{3+} @ \mathrm{NaYF}_{4}: \mathrm{Yb}^{3+}, \mathrm{Nd}^{3+}$ core@shell nanoparticles with the shell layer highly doped with $\mathrm{Nd}^{3+}$ (around $10 \%)^{88}$. Also, doping a high concentration of $\mathrm{Mn}^{2+}$ into $\mathrm{NaYF}_{4}: \mathrm{Yb}^{3+}, \mathrm{Er}^{3+}$ nanocrystals has resulted in pure single-band red upconversion emission via an efficient energy transfer between $\mathrm{Mn}^{2+}$ and $\mathrm{Er}^{3+95}$. Levy et al. used an energy-looping mechanism to non-resonantly excite upconversion in highly $\mathrm{Tm}^{3+}$-doped $\mathrm{NaYF}_{4}: \mathrm{Tm}^{3+}$ nanoparticles with $1064 \mathrm{~nm}$ light for deep-tissue imaging $^{73}$. In this work, as illustrated in Figure $4 \mathrm{c}$, one $\mathrm{Tm}^{3+}$ ion can cross-relax by donating energy partially to a second $\mathrm{Tm}^{3+}$ ion in its ground state, resulting in two $\mathrm{Tm}^{3+}$ ions in their intermediate ${ }^{3} \mathrm{~F}_{4}$ state to enable efficient excited-state absorption at $1064 \mathrm{~nm}$ and emit $800 \mathrm{~nm}$ emissions ${ }^{73}$.

Full-colour/lifetime tuning: The energy transfer between the dopant ions in a core@shell nanostructure has also been found to be controllable by adjusting the pulse duration of the excitation laser (Figure $4 \mathrm{~d}, \mathrm{e})^{63}$. By increasing the pulse duration from 0.2 to $6 \mathrm{~ms}$ (at $980 \mathrm{~nm}$ ), the intensity ratio of green-to-red emission from the shell of $\mathrm{NaYF}_{4}: \mathrm{Yb}^{3+}, \mathrm{Ho}^{3+}, \mathrm{Ce}^{3+}$ with a high concentration of $\mathrm{Ce}^{3+}$ can be continuously modulated. The energy transfer from $\mathrm{Ho}^{3+}$ to $\mathrm{Ce}^{3+}$ by a cross-relaxation process ${ }^{5} \mathrm{I}_{6}\left(\mathrm{Ho}^{3+}\right)+$ ${ }^{2} \mathrm{~F}_{5 / 2}\left(\mathrm{Ce}^{3+}\right) \rightarrow{ }^{5} \mathrm{I}_{7}\left(\mathrm{Ho}^{3+}\right)+{ }^{2} \mathrm{~F}_{7 / 2}\left(\mathrm{Ce}^{3+}\right)$ is only allowed under a long pulse excitation, while the transition from ${ }^{5} \mathrm{I}_{6}\left(\mathrm{Ho}^{3+}\right)$ to higher levels of ${ }^{5} \mathrm{~F}_{4},{ }^{5} \mathrm{~S}_{2}$ prevails over the above cross-relaxation process involving $\mathrm{Ce}^{3+}$ by a short-pulse excitation. This judicious design has further generated pure blue upconversion emission by pumping at $800 \mathrm{~nm}$ by $\mathrm{Nd}^{3+} \rightarrow \mathrm{Yb}^{3+} \rightarrow \mathrm{Tm}^{3+}$ with a high concentration of $\mathrm{Nd}^{3+63}$. Cross-relaxation has been employed for enhancing the downshifting emission between 1500 and 1700 nm for high-spatial resolution and deep-tissue penetration of photons for cerebral vascular image in the second NIR window ${ }^{91}$. Facilitated by the high $\mathrm{Ce}^{3+}$-doping concentration, the $\mathrm{Er}^{3+}{ }^{4} \mathrm{I}_{13 / 2}$ 
level is significantly populated through the accelerated nonradiative relaxation of $\mathrm{Er}^{3+}{ }^{4} \mathrm{I}_{11 / 2} \rightarrow{ }^{4} \mathrm{I}_{13 / 2}$ (Figure $4 \mathrm{f}$ ), resulting in a ninefold enhancement of the downshifting $1550 \mathrm{~nm}$ luminescence of $\mathrm{NaYbF}_{4}: 2 \% \mathrm{Er}^{3+}, 2 \% \mathrm{Ce}^{3+} @ \mathrm{NaYF}_{4}$ nanoparticles.

Apart from colour tuning, luminescence decay lifetimes form another set of optical signatures $79,82,90,100$. Manipulating the degree of cross-relaxation by different $\mathrm{Tm}^{3+}$-doping concentrations can create a large range of lifetimes from 25.6 to $662.4 \mu \mathrm{s}$ in the blue emission band, forming a library of lifetimetunable $\tau$-dots for optical multiplexing (Figure $4 \mathrm{~g})^{90,101}$. Such an optical signature can be used as barcoding for security applications, and only a properly designed time-resolved detector can decode such a set of diverse time-domain optical barcodes. As demonstrated in Figure $4 \mathrm{~h}$, the ability to resolve superimposed

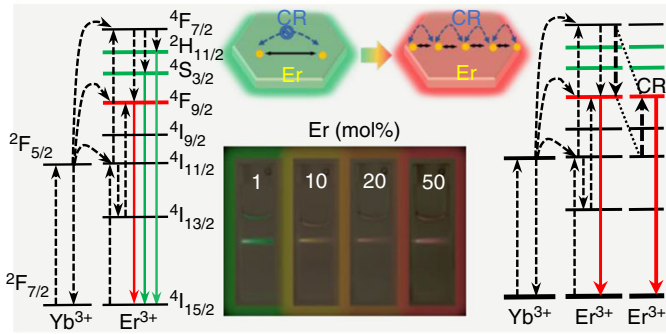

d Short pulse

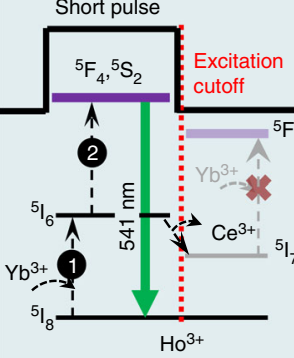

b

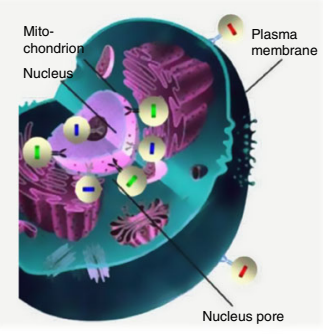

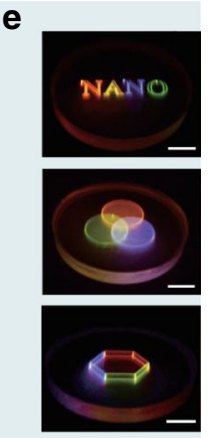

h
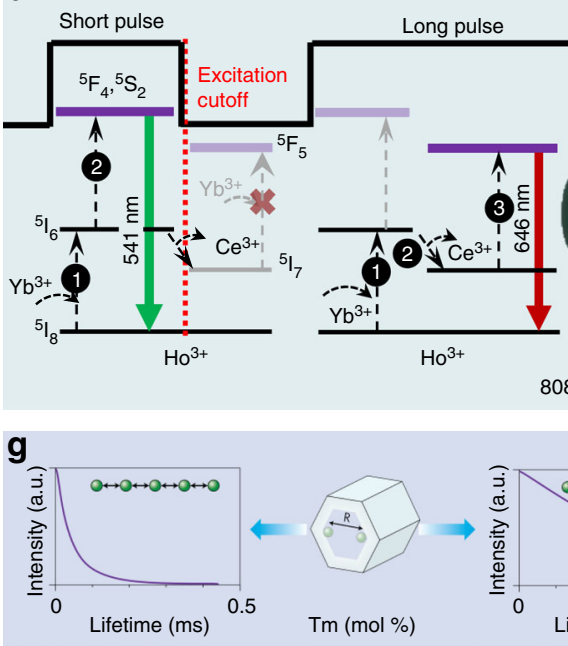

$808 \mathrm{~nm}$ c.w. excitation
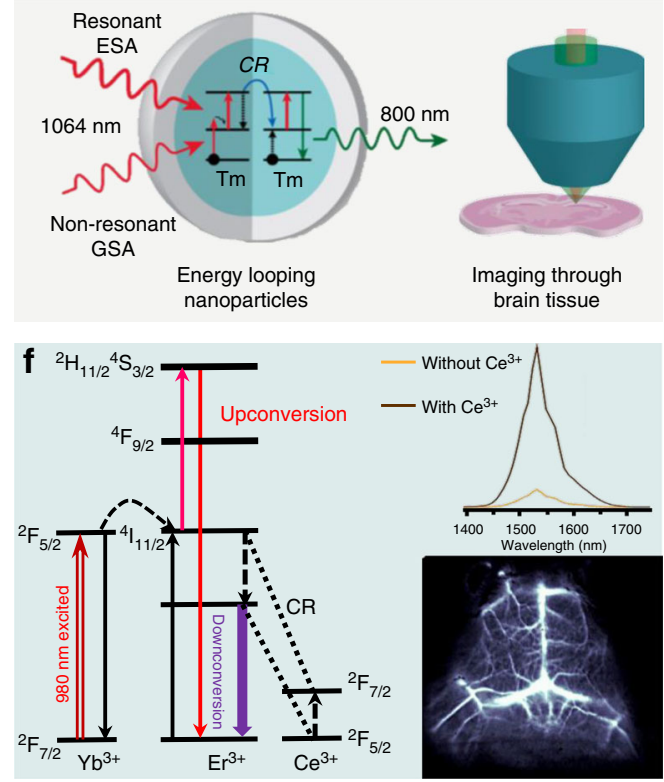

i
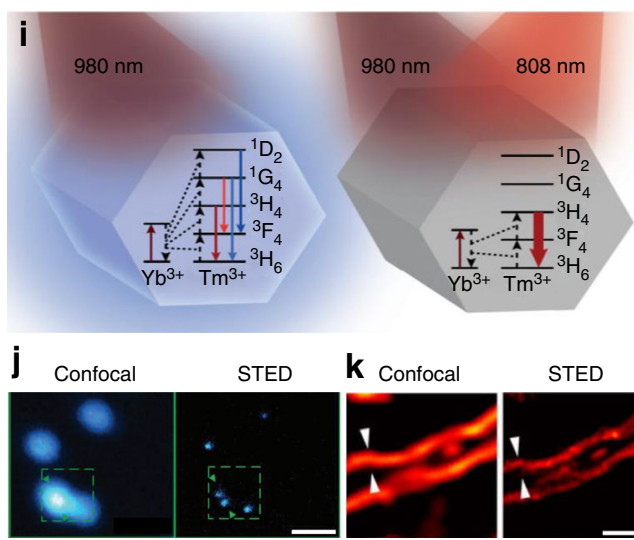

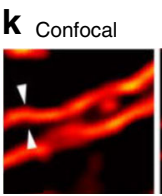

STED

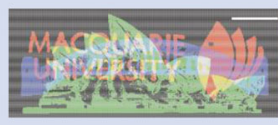

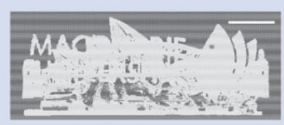

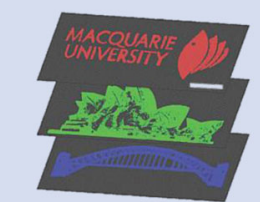

$48.6 \mu$ « $\pm 8.9 \%$

1205

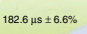

$501.1 \mu \mathrm{s} \pm 8.8 \%$

Lifetime $(\mu \mathrm{s})$

Fig. 4 Cross-relaxation-enabled nanotechnology using highly doped upconversion nanocrystals. a Upconversion mechanisms of Er $3+$ at low- and high-

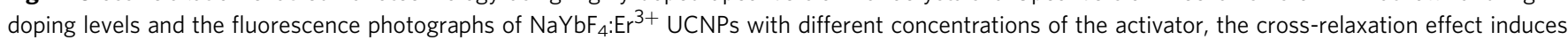

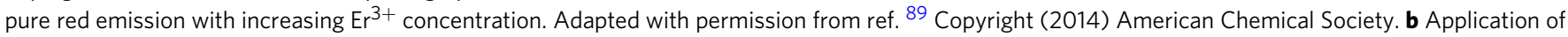
single-band upconversion nanoprobes for multiplexed in situ molecular mapping of cancer biomarkers. Adapted from ref. 93 . c Core@shell design and energy-looping mechanism in highly $\mathrm{Tm}^{3+}$-doped $\mathrm{NaYF}_{4}$, and their application for deep-tissue brain imaging. Reproduced with permission from ref. 73 Copyright (2016) American Chemical Society. d Design and mechanism of NaYF 4 -based core@shell nanocrystals capable of emitting tunable colours

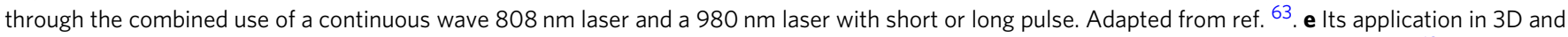
full-colour display systems with high-spatial resolution and locally addressable colour gamut, scale bars represent $1 \mathrm{~cm}$. Adapted from ref. 63 . $\mathbf{f}$ Simplified energy-level diagrams depicting the energy transfer between $\mathrm{Yb}^{3+}, \mathrm{Er}^{3+}$, and $\mathrm{Ce}^{3+}$ ions, downshifting luminescence spectra of the $\mathrm{NaYF}_{4}: \mathrm{Yb}^{3+}, \mathrm{Er}^{3+}$ UCNPs with and without $\mathrm{Ce}^{3+}$ doping and their application for cerebral vascular image through the second NIR window. Adapted from ref. ${ }^{91}$. $\mathbf{g}$ Lifetime tuning scheme and time-resolved confocal images of $\mathrm{NaYF}_{4}: \mathrm{Yb}^{3+}, \mathrm{Tm}^{3+} \mathrm{UCNPs}$ with increasing concentration of Tm ${ }^{3+}$, scale bar represents $1 \mu \mathrm{m}$. Adapted from refs. ${ }^{90,101}$. h Their application in lifetime-encoded document security, scale bars represent $5 \mathrm{~mm}$. Adapted from ref. 90 . i Energy-level diagrams of highly $\mathrm{Tm}^{3+}$-doped UCNPs under $980 \mathrm{~nm}$ and/or $808 \mathrm{~nm}$ illumination. Adapted from ref. ${ }^{1}$. j Confocal versus STED super-resolution images of the 40 nm $8 \% \mathrm{Tm}^{3+}$-doped UCNPs, scale bar represents $500 \mathrm{~nm}$. Adapted from ref. ${ }^{1}$. k Confocal versus STED super-resolution images of cellular cytoskeleton labelled with antibody-conjugated $11.8 \mathrm{~nm} \mathrm{NaGdF} 4: 18 \% \mathrm{Yb}^{3+}, 10 \% \mathrm{Tm}^{3+}$ nanocrystals, scale bar represents $1 \mu \mathrm{m}$. Adapted from ref. 92 
lifetime-encoded images suggests a new way of optical data storage with high densities and fast data readout rates.

Super-resolution imaging: Another intriguing example is nanoscopic imaging using highly $\mathrm{Tm}^{3+}$-doped UCNP as an effective stimulated emission depletion (STED) probe. The advent of super-resolution microscopy, such as STED fluorescence microscopy, has revolutionized biological fluorescence microscopy $^{102,103}$, but STED requires extremely high-power laser densities and specialized fluorescent labels to achieve superresolution imaging. Using the cross-relaxation effect, Liu et al. discovered a photon avalanche effect that facilitates the establishment of population inversion within a single highly $\mathrm{Tm}^{3+}$-doped UCNP (Figure $4 \mathrm{i})^{1}$. This enables sub- $30 \mathrm{~nm}$ optical superresolution imaging with a STED beam density two orders of magnitude lower than that used on fluorescent dyes (Figure $4 \mathrm{j})^{1}$. This effect has only been found in highly doped UCNPs because the cross-relaxation process, dominated at a high $\mathrm{Tm}^{3+}$-doping concentration, can trigger a photon avalanche to establish a population inversion between metastable and ground levels. In that respect, upon $808 \mathrm{~nm}$ beam depletion amplified-stimulated emission is realized, resulting in a higher-depletion efficiency and thus a reduced saturation intensity. Using this new mode of upconversion nanoscopy, Zhan et al. have reported superresolution imaging of cell cytoskeleton with $11.8 \mathrm{~nm}$ $\mathrm{NaGdF}_{4}: 18 \% \mathrm{Yb}^{3+}, 10 \% \mathrm{Tm}^{3+}$ UCNPs (Figure $\left.4 \mathrm{k}\right)^{92}$. Other schemes, based on $\mathrm{Pr}^{3+}$ or $\mathrm{Er}^{3+}$ doped UCNPs, have also been explored for super-resolution nanoscopic applications ${ }^{104,105}$

Perspective. One of the major challenges to transform upconversion nanotechnology into real-world applications is to enhance the brightness and emission efficiency of UCNPs ${ }^{4}$. This review summarizes the advances in the development of highly doped UCNPs and emerging applications by overcoming the concentration quenching effect or smart exploitation of unique features of highly doped nanomaterials. Notably, the unique optical properties arising from the range of layer-by-layer heterogeneously doped nanoparticles have attracted immense scientific and technological interests. The intentional doping of high concentration of lanthanide ions into different sections across a single UCNP has been explored to enhance the desirable optical properties as well as introducing multifunctionality. Thus far, only spherical core@shell structures have been studied to modulate the energy transfer, while further investigations of heterogeneous one-dimension structures, such as rods, plates and dumbbells, are still needed ${ }^{60,106,107}$. Controlled growth toward atomic precision is highly sought after for gaining a full understanding of the sophisticated energy transfer processes and for fine-tuning upconversion luminescence. For example, arranging high concentrations of dopants into a host nanocrystal along one direction could confine the direction of energy transfer, which may create new properties and enable novel applications going beyond the current isotropic $3 \mathrm{D}$ transfer processes.

The unique optical properties of highly doped UCNPs discussed above have largely impacted biological and biomedical fields, such as single-molecule sensing ${ }^{18}$, high-throughput multiplexed detection ${ }^{79,82,90}$ and super-resolution nanoscopy ${ }^{1,92}$. It is noteworthy that small-sized and bright UCNPs are indispensable to those applications. Owing to brightness issues, the majority of currently developed UCNPs are relatively large (around 20-50 $\mathrm{nm}$ ). It has been challenging to design and fabricate highly doped sub-10 nm UCNPs with emission output comparable with that of quantum dots and organic dyes. To our delight, fine-tuning of the particle size below $10 \mathrm{~nm}$ was recently demonstrated for UCNP systems by homogeneous doping ${ }^{108}$ or at a high-doping concentrations ${ }^{109}$. Nonetheless, the fabrication of sub- $10 \mathrm{~nm}$
UCNPs with heterogeneously doped core@shell structures remains a formidable challenge.

The surface molecules not only play an essential role in the controlled synthesis of nanomaterials, but they can also significantly alter the nanomaterial's luminescence properties with new effects ${ }^{110,111}$. Examples include the recent developments of dye-sensitized UCNPs ${ }^{16,62,112,113}$ and surface phonon enhanced UCNPs in a thermal field ${ }^{111}$. Normally, lanthanidedoped inorganic nanocrystals exhibit narrowband (FWHM around $20 \mathrm{~nm})$ and low $\left(10^{-20} \mathrm{~cm}^{-2}\right)$ absorption coefficients. It is notable that organic dyes have more than 10 times broader absorption spectra and $10^{3}-10^{4}$-fold higher absorption crosssections than $\mathrm{Yb}^{3+}$ sensitizer ions commonly used in $\mathrm{UCNPs}^{16,62,112,113}$. Therefore, despite photostability issues, the organic-inorganic hybrid nanomaterials (for example, dyesensitized upconversion nanosystems) offer various possibilities $^{16}$. Utilizing the efficient energy transfer of cyanine derivatives anchored on the surface of $\mathrm{NaYbF}_{4}: \mathrm{Tm}^{3+} @ \mathrm{NaYF}_{4}: \mathrm{Nd}^{3+}$ nanoparticles, Chen et al. demonstrated dye-sensitized upconver$\operatorname{sion}^{16}$. Upon $800 \mathrm{~nm}$ excitation, a sequential energy transfer, dye $\rightarrow \mathrm{Nd}^{3+} \rightarrow \mathrm{Yb}^{3+} \rightarrow$ activators, has enabled the dye-sensitized nanoparticles to emit around 25 times stronger than canonical $\mathrm{NaYF}_{4}: \mathrm{Yb}^{3+}, \mathrm{Tm}^{3+} @ \mathrm{NaYF}_{4}$ nanoparticles excited at $980 \mathrm{~nm}$. Similarly, the common triplet energy transfer could occur between inorganic nanocrystals and the surface dyes ${ }^{114-118}$. While thermal quenching broadly limits the luminescence efficiency at high temperatures in optical materials, Zhou et al. report that the phonons at the surface of highly $\mathrm{Yb}^{3+}$-doped UCNPs could combat thermal quenching and significantly enhance the upconversion brightness, particularly for sub-10 $\mathrm{nm}$ nanocrystals ${ }^{111}$. We believe that these hybrid and heterogeneously doped nanomaterials have the potential in pushing the performance of UCNPs to a new level and imparting multifaceted photonic applications.

Received: 4 December 2017 Accepted: 25 May 2018

Published online: 20 June 2018

\section{References}

1. Liu, Y. et al. Amplified stimulated emission in upconversion nanoparticles for super-resolution nanoscopy. Nature 543, 229-233 (2017).

This paper presents the discovery of amplified stimulated emission in highly doped UCNPs and the application to realize super resolution (28 $\mathrm{nm}$ ) optical nanoscopy using low power diode lasers.

2. Zhou, B., Shi, B., Jin, D. \& Liu, X. Controlling upconversion nanocrystals for emerging applications. Nat. Nanotechnol. 10, 924-936 (2015).

3. Wang, F. et al. Simultaneous phase and size control of upconversion nanocrystals through lanthanide doping. Nature 463, 1061-1065 (2010).

4. Wilhelm, S. Perspectives for upconverting nanoparticles. ACS Nano 11, 10644-10653 (2017)

5. Chan, E. M. Combinatorial approaches for developing upconverting nanomaterials: high-throughput screening, modeling, and applications. Chem. Soc. Rev. 44, 1653-1679 (2015).

6. Liu, X., Yan, C.-H. \& Capobianco, J. A. Photon upconversion nanomaterials. Chem. Soc. Rev. 44, 1299-1301 (2015).

7. Tu, L., Liu, X., Wu, F. \& Zhang, H. Excitation energy migration dynamics in upconversion nanomaterials. Chem. Soc. Rev. 44, 1331-1345 (2015).

8. Chen, X., Peng, D., Ju, Q. \& Wang, F. Photon upconversion in core-shell nanoparticles. Chem. Soc. Rev. 44, 1318-1330 (2015).

9. Yang, P., Deng, P. \& Yin, Z. Concentration quenching in Yb:YAG. J. Lumin 97, 51-54 (2002).

10. Viger, M. L., Live, L. S., Therrien, O. D. \& Boudreau, D. Reduction of selfquenching in fluorescent silica-coated silver nanoparticles. Plasmonics 3, 33-40 (2008).

11. Tavernaro, I., Cavelius, C., Peuschel, H. \& Kraegeloh, A. Bright fluorescent silica-nanoparticle probes for high-resolution STED and confocal microscopy. Beilstein J. Nanotechnol. 8, 1283-1296 (2017). 
12. Danielmeyer, H. G., Blätte, M. \& Balmer, P. Fluorescence quenching in Nd: YAG. Appl. Phys. 1, 269-274 (1973).

13. Haase, M. \& Schäfer, H. Upconverting nanoparticles. Angew. Chem. Int. Ed. 50, 5808-5829 (2011).

14. Boyer, J.-C. \& van Veggel, F. C. J. M. Absolute quantum yield measurements of colloidal NaYF4: $\mathrm{Er}^{3+}, \mathrm{Yb}^{3+}$ upconverting nanoparticles. Nanoscale 2, 1417-1419 (2010).

15. Wang, F., Wang, J. \& Liu, X. Direct evidence of a surface quenching effect on size-dependent luminescence of upconversion nanoparticles. Angew. Chem. Int. Ed. 49, 7456-7460 (2010).

16. Chen, G. et al. Energy-cascaded upconversion in an organic dye-sensitized core/shell fluoride nanocrystal. Nano Lett. 15, 7400-7407 (2015).

17. Zhao, J. et al. Single-nanocrystal sensitivity achieved by enhanced upconversion luminescence. Nat. Nanotechnol. 8, 729-734 (2013). This paper is the first report of ultra-bright highly $\mathrm{Tm}^{3+}$ doped UCNPs under condensed excitation power.

18. Gargas, D. J. et al. Engineering bright sub-10-nm upconverting nanocrystals for single-molecule imaging. Nat. Nanotechnol. 9, 300-305 (2014). This paper reports the high brightness of sub-10 $\mathrm{nm}$ highly $\mathrm{Er}^{3+}$ doped UCNPs under condesned excitation power.

19. Dexter, D. L. \& Schulman, J. H. Theory of concentration quenching in inorganic phosphors. J. Chem. Phys. 22, 1063-1070 (1954).

20. Chen, R. F. \& Knutson, J. R. Mechanism of fluorescence concentration quenching of carboxyfluorescein in liposomes: energy transfer to nonfluorescent dimers. Anal. Biochem. 172, 61-77 (1988).

21. Arbeloa, I. L. Dimeric and trimeric states of the fluorescein dianion. Part 2.Effects on fluorescence characteristics. J. Chem. Soc. Faraday Trans. 2, 1735-1742 (1981).

22. Zhao, G.-J., Liu, J.-Y., Zhou, L.-C. \& Han, K.-L. Site-selective photoinduced electron transfer from alcoholic solvents to the chromophore facilitated by hydrogen bonding: a new fluorescence quenching mechanism. J. Phys. Chem. B 111, 8940-8945 (2007)

23. Imhof, A. et al. Spectroscopy of fluorescein (FITC) dyed colloidal silica spheres. J. Phys. Chem. B 103, 1408-1415 (1999).

24. Wang, J. et al. Enhancing multiphoton upconversion through energy clustering at sublattice level. Nat. Mater. 13, 157-162 (2014).

25. Johnson, N. J. J. et al. Direct evidence for coupled surface and concentration quenching dynamics in lanthanide-doped nanocrystals. J. Am. Chem. Soc. 139, 3275-3282 (2017).

This paper provides direct evidence that the energy migration to the surface causes the quenching of highy doped nanoparticles.

26. Jares-Erijman, E. A. \& Jovin, T. M. FRET imaging. Nat. Biotechnol. 21, 1387-1395 (2003).

27. Sun, T., Ma, R., Qiao, X., Fan, X. \& Wang, F. Shielding upconversion by surface coating: a study of the emission enhancement factor. Chemphyschem 17, 766-770 (2016)

28. Wang, F. \& Liu, X. Upconversion multicolor fine-tuning: visible to nearinfrared emission from lanthanide-doped NaYF4 nanoparticles. J. Am. Chem. Soc. 130, 5642-5643 (2008).

29. Heer, S., Kömpe, K., Güdel, H. U. \& Haase, M. Highly efficient multicolour upconversion emission in transparent colloids of lanthanide-doped NaYF4 nanocrystals. Adv. Mater. 16, 2102-2105 (2004).

30. Kawamura, Y., Brooks, J., Brown, J. J., Sasabe, H. \& Adachi, C. Intermolecular interaction and a concentration-quenching mechanism of phosphorescent Ir (III) complexes in a solid film. Phys. Rev. Lett. 96, 017404 (2006).

31. Nadort, A., Zhao, J. \& Goldys, E. M. Lanthanide upconversion luminescence at the nanoscale: fundamentals and optical properties. Nanoscale $\mathbf{8}$, 13099-13130 (2016).

32. Auzel, F. Upconversion and anti-stokes processes with $\mathrm{f}$ and $\mathrm{d}$ ions in solids. Chem. Rev. 104, 139-174 (2004).

33. Qin, X., Liu, X., Huang, W., Bettinelli, M. \& Liu, X. Lanthanide-activated phosphors based on $4 \mathrm{f}-5 \mathrm{~d}$ optical transitions: theoretical and experimental aspects. Chem. Rev. 117, 4488-4527 (2017).

34. Wang, F. et al. Tuning upconversion through energy migration in core-shell nanoparticles. Nat. Mater. 10, 968-973 (2011).

This paper discovers the $\mathrm{Gd}^{3+}$-mediated energy migration and demonstrates the highly $\mathrm{Tb}^{3+}$ and $E u^{3+}$ doped core@shell@shell nanocrystals for the fine tuning of upconversion emission colours.

35. Wang, Y.-F. et al. $\mathrm{Nd}^{3+}$-sensitized upconversion nanophosphors: efficien in vivo bioimaging probes with minimized heating effect. ACS Nano 7 , 7200-7206 (2013).

This paper reports that the heterogenously highly $\mathrm{Nd}^{3+}$ doped core@shell nanomaterials emit significantly enhanced upconversion luminescence compared to the homogeneously doped nanocrystals.

36. Zuo, J. et al. Employing shells to eliminate concentration quenching in photonic upconversion nanostructure. Nanoscale 9, 7941-7946 (2017).

37. Benz, F. \& Strunk, H. P. Rare earth luminescence: a way to overcome concentration quenching. AIP Adv. 2, 042115 (2012)
38. Marciniak, L., Strek, W., Bednarkiewicz, A., Lukowiak, A. \& Hreniak, D Bright upconversion emission of $\mathrm{Nd}^{3+}$ in LiLa1-xNdxP4O12 nanocrystalline powders. Opt. Mater. 33, 1492-1494 (2011).

39. $\mathrm{Xu}, \mathrm{X}$. et al. Depth-profiling of $\mathrm{Yb}^{3+}$ sensitizer ions in NaYF4 upconversion nanoparticles. Nanoscale 9, 7719-7726 (2017).

40. Pilch, A. et al. Shaping luminescent properties of $\mathrm{Yb}^{3+}$ and $\mathrm{Ho}^{3+} \mathrm{Co}$-doped upconverting core-shell $\beta$-NaYF4 nanoparticles by dopant distribution and spacing. Small 13, 1701635 (2017).

41. Liu, X. et al. Breakthrough in concentration quenching threshold of upconversion luminescence via spatial separation of the emitter doping area for bio-applications. Chem. Commun. 47, 11957-11959 (2011).

42. Jin, D. \& Zhao, J. Enhancing upconversion luminescence in rare-earth doped particles. US patent US20150252259A1 (2015).

43. Li, X., Wang, R., Zhang, F. \& Zhao, D. Engineering homogeneous doping in single nanoparticle to enhance upconversion efficiency. Nano Lett. 14, 3634-3639 (2014)

44. Wang, F. et al. Microscopic inspection and tracking of single upconversion nanoparticles in living cells. Light Sci. Appl. 7, e18007 (2018).

45. Wei, W. et al. Alleviating luminescence concentration quenching in upconversion nanoparticles through organic dye sensitization. J. Am. Chem. Soc. 138, 15130-15133 (2016).

46. Chen, G., Ohulchanskyy, T. Y., Kumar, R., Ågren, H. \& Prasad, P. N. Ultrasmall monodisperse NaYF4:Yb ${ }^{3+} / \mathrm{Tm}^{3+}$ nanocrystals with enhanced near-infrared to near-infrared upconversion photoluminescence. ACS Nano 4 , 3163-3168 (2010)

47. Shen, B. et al. Revisiting the optimized doping ratio in core/shell nanostructured upconversion particles. Nanoscale 9, 1964-1971 (2017).

48. Chen, G. et al. $\left(\alpha-\mathrm{NaYbF} 4: \mathrm{Tm}^{3+}\right) / \mathrm{CaF} 2$ core/shell nanoparticles with efficient near-infrared to near-infrared upconversion for high-contrast deep tissue bioimaging. ACS Nano 6, 8280-8287 (2012).

49. Punjabi, A. et al. Amplifying the red-emission of upconverting nanoparticles for biocompatible clinically used prodrug-induced photodynamic therapy. ACS Nano 8, 10621-10630 (2014).

50. Shen, J. et al. Tunable near infrared to ultraviolet upconversion luminescence enhancement in ( $\alpha$-NaYF4:Yb,Tm)/CaF2 core/shell nanoparticles for in situ real-time recorded biocompatible photoactivation. Small 9, 3213-3217 (2013).

51. Xue, M. et al. Highly enhanced cooperative upconversion luminescence through energy transfer optimization and quenching protection. ACS Appl. Mater. Interfaces 8, 17894-17901 (2016).

52. Ma, C. et al. Optimal sensitizer concentration in single upconversion nanocrystals. Nano Lett. 17, 2858-2864 (2017).

53. Zhang, Y. \& Liu, X. Nanocrystals: shining a light on upconversion. Nat. Nanotechnol. 8, 702-703 (2013)

54. Li, Y. et al. Enhancing upconversion fluorescence with a natural biomicrolens. ACS Nano 11, 10672-10680 (2017).

55. Wang, L. et al. Reversible near-infrared light directed reflection in a selforganized helical superstructure loaded with upconversion nanoparticles. J. Am. Chem. Soc. 136, 4480-4483 (2014).

56. Chen, X. et al. Confining energy migration in upconversion nanoparticles towards deep ultraviolet lasing. Nat. Commun. 7, 10304 (2016).

57. Drees, C. et al. Engineered upconversion nanoparticles for resolving protein interactions inside living cells. Angew. Chem. Int. Ed. 55, 11668-11672 (2016).

This paper employs the high brightness of highly doped UCNPs to study the protein Interactions inside living cells.

58. Pliss, A. et al. Subcellular optogenetics enacted by targeted nanotransformers of near-infrared light. ACS Photonics 4, 806-814 (2017).

59. Kovalenko, M. V. et al. Prospects of nanoscience with nanocrystals. ACS Nano 9, 1012-1057 (2015).

60. Liu, D. et al. Three-dimensional controlled growth of monodisperse sub-50 $\mathrm{nm}$ heterogeneous nanocrystals. Nat. Commun. 7, 10254 (2016).

61. Xie, X. et al. Mechanistic investigation of photon upconversion in $\mathrm{Nd}^{3+}$-sensitized core-shell nanoparticles. J. Am. Chem. Soc. 135 12608-12611 (2013).

62. Chen, G. et al. Efficient broadband upconversion of near-infrared light in dyesensitized core/shell nanocrystals. Adv. Opt. Mater. 4, 1760-1766 (2016)

63. Deng, R. et al. Temporal full-colour tuning through non-steady-state upconversion. Nat. Nanotechnol. 10, 237-242 (2015).

64. Hao, S. et al. $\mathrm{Nd}^{3+}$-sensitized multicolor upconversion luminescence from a sandwiched core/shell/shell nanostructure. Nanoscale 9, 10633-10638 (2017).

65. Marciniak, L., Pilch, A., Arabasz, S., Jin, D. \& Bednarkiewicz, A. Heterogeneously $\mathrm{Nd}^{3+}$ doped single nanoparticles for NIR-induced heat conversion, luminescence, and thermometry. Nanoscale 9, 8288-8297 (2017).

66. Chen, X. et al. Energy migration upconversion in Ce(III)-doped heterogeneous core-shell-shell nanoparticles. Small 13, 1701479 (2017).

67. Zhang, C. et al. White-light emission from an integrated upconversion nanostructure: toward multicolor displays modulated by laser power. Angew. Chem. Int. Ed. 54, 11531-11535 (2015). 
68. Deng, R., Wang, J., Chen, R., Huang, W. \& Liu, X. Enabling Förster resonance energy transfer from large nanocrystals through energy migration. J. Am. Chem. Soc. 138, 15972-15979 (2016).

69. Li, X. et al. Energy migration upconversion in manganese(II)-doped nanoparticles. Angew. Chem. Int. Ed. 54, 13312-13317 (2015).

70. Zhou, B., Yang, W., Han, S., Sun, Q. \& Liu, X. Photon upconversion through $\mathrm{Tb}^{3+}$-mediated interfacial energy transfer. Adv. Mater. 27, 6208-6212 (2015).

71. Nam, S. H. et al. Long-term real-time tracking of lanthanide ion doped upconverting nanoparticles in living cells. Angew. Chem. Int. Ed. 50, 6093-6097 (2011).

72. Xie, $X$. et al. Emerging $\approx 800 \mathrm{~nm}$ excited lanthanide-doped upconversion nanoparticles. Small 13, 1602843 (2017).

73. Levy, E. S. et al. Energy-looping nanoparticles: harnessing excited-state absorption for deep-tissue imaging. ACS Nano 10, 8423-8433 (2016). This paper employs energy-looping mechanism to enable the intermediate excited-state absorption at 1064nm for highly $\mathrm{Tm}^{3+}$-doped UCNPs.

74. Zhong, Y. et al. Elimination of photon quenching by a transition layer to fabricate a quenching-shield sandwich structure for $800 \mathrm{~nm}$ excited upconversion luminescence of $\mathrm{Nd}^{3+}$-sensitized nanoparticles. Adv. Mater. 26, 2831-2837 (2014).

75. Zhong, Y., Rostami, I., Wang, Z., Dai, H. \& Hu, Z. Energy migration engineering of bright rare-earth upconversion nanoparticles for excitation by light-emitting diodes. Adv. Mater. 27, 6418-6422 (2015).

76. Prorok, K., Pawlyta, M., Stręk, W. \& Bednarkiewicz, A. Energy migration upconversion of $\mathrm{Tb}^{3+}$ in $\mathrm{Yb}^{3+}$ and $\mathrm{Nd}^{3+}$ codoped active-core/active-shell colloidal nanoparticles. Chem. Mater. 28, 2295-2300 (2016).

77. Marciniak, L., Prorok, K., Frances-Soriano, L., Perez-Prieto, J. \& Bednarkiewicz, A. A broadening temperature sensitivity range with a coreshell YbEr@YbNd double ratiometric optical nanothermometer. Nanoscale 8, 5037-5042 (2016).

78. Wen, $\mathrm{H}$. et al. Upconverting near-infrared light through energy management in core-shell-shell nanoparticles. Angew. Chem. Int. Ed. 52, 13419-13423 (2013).

79. Liu, X. et al. Binary temporal upconversion codes of $\mathrm{Mn}^{3+}$-activated nanoparticles for multilevel anti-counterfeiting. Nat. Commun. 8, 899 (2017).

80. Lai, J., Zhang, Y., Pasquale, N. \& Lee, K.-B. An upconversion nanoparticle with orthogonal emissions using dual nir excitations for controlled two-way photoswitching. Angew. Chem. Int. Ed. 53, 14419-14423 (2014).

81. Li, X. et al. Filtration shell mediated power density independent orthogonal excitations-emissions upconversion luminescence. Angew. Chem. Int. Ed. 55, 2464-2469 (2016).

82. Dong, H. et al. Versatile spectral and lifetime multiplexing nanoplatform with excitation orthogonalized upconversion luminescence. ACS Nano 11, 3289-3297 (2017).

83. $\mathrm{Li}, \mathrm{X}$. et al. $\mathrm{Nd}^{3+}$ sensitized up/down converting dual-mode nanomaterials for efficient in-vitro and in-vivo bioimaging excited at 800nm. Sci. Rep. 3, 3536 (2013).

84. He, S. et al. Simultaneous enhancement of photoluminescence, MRI relaxivity, and CT contrast by tuning the interfacial layer of lanthanide heteroepitaxial nanoparticles. Nano Lett. 17, 4873-4880 (2017).

85. Wang, L. et al. Luminescence-driven reversible handedness inversion of selforganized helical superstructures enabled by a novel near-infrared light nanotransducer. Adv. Mater. 27, 2065-2069 (2015).

86. Shao, Q. et al. Emission color tuning of core/shell upconversion nanoparticles through modulation of laser power or temperature. Nanoscale 9, 12132-12141 (2017).

87. $\mathrm{Li}, \mathrm{Y}$. et al. A versatile imaging and therapeutic platform based on dual-band luminescent lanthanide nanoparticles toward tumor metastasis inhibition. ACS Nano 10, 2766-2773 (2016).

88. Chen, D. et al. $\mathrm{Nd}^{3+}$-sensitized $\mathrm{Ho}^{3+}$ single-band red upconversion luminescence in core-shell nanoarchitecture. J. Phys. Chem. Lett. 6, 2833-2840 (2015).

89. Wei, W. et al. Cross relaxation induced pure red upconversion in activator and sensitizer-rich lanthanide nanoparticles. Chem. Mater. 26, 5183-5186 (2014).

90. Lu, Y. et al. Tunable lifetime multiplexing using luminescent nanocrystals. Nat. Photon. 8, 32-36 (2014).

This paper employs the large dynamic range of $\mathrm{Tm}^{3+}$ doping concentrations to create a large dynamic range of luminescence decay lifetimes and to build a library of lifetime-tunable $\tau$-dots for optical multiplexing.

91. Zhong, Y. et al. Boosting the downshifting luminescence of rare-earth nanocrystals for biological imaging beyond 1500nm. Nat. Commun. 8, 737 (2017).

92. Zhan, Q. et al. Achieving high-efficiency emission depletion nanoscopy by employing cross relaxation in upconversion nanoparticles. Nat. Commun. 8, 1058 (2017).
93. Zhou, L. et al. Single-band upconversion nanoprobes for multiplexed simultaneous in situ molecular mapping of cancer biomarkers. Nat. Commun. 6, 6938 (2015).

94. Wang, J., Wang, F., Wang, C., Liu, Z. \& Liu, X. Single-band upconversion emission in lanthanide-doped KMnF3 nanocrystals. Angew. Chem. Int. Ed. 50 10369-10372 (2011).

95. Tian, G. et al. $\mathrm{Mn}^{3+}$ dopant-controlled synthesis of NaYF4:Yb/Er upconversion nanoparticles for in vivo imaging and drug delivery. Adv. Mater. 24, 1226-1231 (2012).

96. Chan, E. M. et al. Combinatorial discovery of lanthanide-doped nanocrystals with spectrally pure upconverted emission. Nano Lett. 12, 3839-3845 (2012).

97. Chen, Q. et al. Confining excitation energy in $\mathrm{Er}^{3+}$-sensitized upconversion nanocrystals through $\mathrm{Tm}^{3+}$-mediated transient energy trapping. Angew. Chem. Int. Ed. 56, 7605-7609 (2017).

98. Chen, G., Liu, H., Somesfalean, G., Liang, H. \& Zhang, Z. Upconversion emission tuning from green to red in $\mathrm{Yb}^{3+} / \mathrm{Ho}^{3+}{ }^{3+}$-codoped NaYF 4 nanocrystals by tridoping with $\mathrm{Ce}^{3+}$ ions. Nanotechnology 20, 385704 (2009).

99. Gao, W. et al. Enhanced red upconversion luminescence by codoping $\mathrm{Ce}^{3+}$ in [small beta]-NaY(Gd0.4)F4: $\mathrm{Yb}^{3+} / \mathrm{Ho}^{3+}$ nanocrystals. J. Mater. Chem. C 2, 5327-5334 (2014).

100. Zhao, J. et al. Upconversion luminescence with tunable lifetime in NaYF4:Yb, Er nanocrystals: role of nanocrystal size. Nanoscale 5, 944-952 (2013).

101. Deng, R. \& Liu, X. Optical multiplexing: tunable lifetime nanocrystals. Nat. Photon. 8, 10-12 (2013).

102. Balzarotti, F. et al. Nanometer resolution imaging and tracking of fluorescent molecules with minimal photon fluxes. Science 355, 606-612 (2016).

103. Hanne, J. et al. STED nanoscopy with fluorescent quantum dots. Nat. Commun. 6, 7127 (2015).

104. Shin, K. et al. Distinct mechanisms for the upconversion of NaYF4: $\mathrm{Yb}^{3+}$, $\mathrm{Er}^{3+}$ nanoparticles revealed by stimulated emission depletion. Phys. Chem. Chem. Phys. 19, 9739-9744 (2017).

105. Kolesov, R. et al. Super-resolution upconversion microscopy of praseodymium-doped yttrium aluminum garnet nanoparticles. Phys. Rev. B 84, 153413 (2011)

106. Zhuo, Z. et al. Manipulating energy transfer in lanthanide-doped single nanoparticles for highly enhanced upconverting luminescence. Chem. Sci. 8, 5050-5056 (2017).

107. Wen, H.-Q. et al. Sequential growth of NaYF4:Yb/Er@NaGdF4 nanodumbbells for dual-modality fluorescence and magnetic resonance imaging. ACS Appl. Mater. Interfaces 9, 9226-9232 (2017).

108. Rinkel, T., Raj, A. N., Dühnen, S. \& Haase, M. Synthesis of 10nm $\beta-N a Y F 4: Y b$, $\mathrm{Er} / \mathrm{NaYF} 4$ core/shell upconversion nanocrystals with $5 \mathrm{~nm}$ particle cores. Angew. Chem. Int. Ed. 55, 1164-1167 (2016).

109. Shi, R. et al. Tuning hexagonal NaYbF4 nanocrystals down to sub-10 nm for enhanced photon upconversion. Nanoscale 9, 13739-13746 (2017).

110. Boles, M. A., Ling, D., Hyeon, T. \& Talapin, D. V. The surface science of nanocrystals. Nat. Mater. 15, 141-153 (2016).

111. Zhou, J. et al. Activation of the surface dark-layer to enhance upconversion in a thermal field. Nat. Photon. 12, 154-158 (2018).

112. $\mathrm{Wu}, \mathrm{X}$. et al. Tailoring dye-sensitized upconversion nanoparticle excitation bands towards excitation wavelength selective imaging. Nanoscale 7 , 18424-18428 (2015).

113. $\mathrm{Wu}, \mathrm{X}$. et al. Dye-sensitized core/active shell upconversion nanoparticles for optogenetics and bioimaging applications. ACS Nano 10, 1060-1066 (2016).

114. Mongin, C., Garakyaraghi, S., Razgoniaeva, N., Zamkov, M. \& Castellano, F. N. Direct observation of triplet energy transfer from semiconductor nanocrystals. Science 351, 369-372 (2016).

115. Wu, M. et al. Solid-state infrared-to-visible upconversion sensitized by colloidal nanocrystals. Nat. Photon. 10, 31-34 (2016).

116. Tabachnyk, M. et al. Resonant energy transfer of triplet excitons from pentacene to PbSe nanocrystals. Nat. Mater. 13, 1033-1038 (2014).

117. Huang, Z. et al. Hybrid molecule-nanocrystal photon upconversion across the visible and near-infrared. Nano Lett. 15, 5552-5557 (2015).

118. Garfield, D. J. et al. Enrichment of molecular antenna triplets amplifies upconverting nanoparticle emission. Nat. Photon. https://doi.org/10.1038/ s41566-018-0156-x (2018).

\section{Acknowledgements}

This project is primarily supported by the Australian Research Council (ARC) Future Fellowship Scheme (D.J., FT130100517), ARC Discovery Early Career Researcher Award Scheme (J.Z., DE180100669), National Natural Science Foundation of China (NSFC, 61729501, 51720105015), Singapore Ministry of Education (Grant R143000627112 and R143000642112), National Research Foundation, Prime Minister's Office, Singapore under its Competitive Research Program (CRP Award No. NRF-CRP15-2015-03), and the European Upconversion Network (COST Action CM1403). 


\section{Author contributions}

S.W. and D.J. were responsible for structuring and drafting the manuscript; J.Z. developed the boxes; S.W., J.Z., and K.Z. developed the figures; and S.W., J.Z., K.Z., A.B., X.L., and D.J. edited and revised the manuscript.

\section{Additional information}

Competing interests: The authors declare no competing interests.

Reprints and permission information is available online at http://npg.nature.com/ reprintsandpermissions/

Publisher's note: Springer Nature remains neutral with regard to jurisdictional claims in published maps and institutional affiliations. (c) (i) Open Access This article is licensed under a Creative Commons Attribution 4.0 International License, which permits use, sharing, adaptation, distribution and reproduction in any medium or format, as long as you give appropriate credit to the original author(s) and the source, provide a link to the Creative Commons license, and indicate if changes were made. The images or other third party material in this article are included in the article's Creative Commons license, unless indicated otherwise in a credit line to the material. If material is not included in the article's Creative Commons license and your intended use is not permitted by statutory regulation or exceeds the permitted use, you will need to obtain permission directly from the copyright holder. To view a copy of this license, visit http://creativecommons.org/ licenses/by/4.0/.

(C) The Author(s) 2018 